\title{
Taking advantage of the software product line paradigm to generate customized user interfaces for decision-making processes: a case study on university employability
}

\author{
Andrea Vázquez-Ingelmo ${ }^{\text {Corresp., } 1}$, Francisco J García-Peñalvo ${ }^{1}$, Roberto Therón Sánchez ${ }^{1,2}$ \\ ${ }^{1}$ GRIAL Research Group, Department of Computer Science and Automatics, University of Salamanca, Salamanca, Spain \\ 2 VisUSAL Research Group, Department of Computer Science and Automatics, University of Salamanca, Salamanca, Spain \\ Corresponding Author: Andrea Vázquez-Ingelmo \\ Email address: andreavazquez@usal.es
}

University employment and, specifically, employability has gained relevance since research in these fields can lead to improvement in the quality of life of individual citizens. However, empirical research is still insufficient to make significant decisions, and relying on powerful tools to explore data and reach insights on these fields is paramount. Information dashboards play a key role in analyzing and visually exploring data about a specific topic or domain, but end users can present several necessities that differ from each other, regarding the displayed information itself, design features and even functionalities. By applying a domain engineering approach (within the software product line paradigm), it is possible to produce customized dashboards to fit into particular requirements, by the identification of commonalities and singularities of every product that could be part of the product line. Software product lines increase productivity, maintainability and traceability regarding the evolution of the requirements, among other benefits. To validate this approach, a case study of its application in the context of the Spanish Observatory for University Employability and Employment system has been developed, where users (Spanish universities and administrators) can control their own dashboards to reach insights about the employability of their graduates. These dashboards have been automatically generated through a domain specific language, which provides the syntax to specify the requirements of each user. The domain language fuels a template-based code generator, allowing the generation of the dashboards' source code. Applying domain engineering to the dashboards' domain improves the development and maintainability of these complex software products given the variety of requirements that users might have regarding their graphical interfaces. 
1 Taking advantage of the software product line

2 paradigm to generate customized user interfaces for

3 decision-making processes: a case study on

4 university employability

5

6 Andrea Vázquez-Ingelmo ${ }^{1}$, Francisco J. García-Peñalvo ${ }^{1}$, Roberto Therón ${ }^{1,2}$

7

$8{ }^{1}$ GRIAL Research Group. Department of Computer Science and Automatics, University of

9 Salamanca, Salamanca, Salamanca, Spain

$10{ }^{2}$ VisUSAL Research Group. Department of Computer Science and Automatics, University of

11 Salamanca, Salamanca, Salamanca, Spain

12

13 Corresponding Author:

14 Andrea Vázquez-Ingelmo ${ }^{1}$

15 Paseo de Canalejas 169, Salamanca, Salamanca, 37008, Spain

16 Email address: andreavazquez@usal.es 
18

19

20

21

22

23

24

25

26

27

28

29

30

31

32

33

34

35

36

37

38

39

40

41

42

43

44

45

46

47

48

49

50

51

52

53

54

55

56

57

\section{ABSTRACT}

University employment and, specifically, employability has gained relevance since research in these fields can lead to improvement in the quality of life of individual citizens. However, empirical research is still insufficient to make significant decisions, and relying on powerful tools to explore data and reach insights on these fields is paramount. Information dashboards play a key role in analyzing and visually exploring data about a specific topic or domain, but end users can present several necessities that differ from each other, regarding the displayed information itself, design features, and even functionalities. By applying a domain engineering approach (within the software product line paradigm), it is possible to produce customized dashboards to fit into particular requirements, by the identification of commonalities and singularities of every product that could be part of the product line. Software product lines increase productivity, maintainability and traceability regarding the evolution of the requirements, among other benefits. To validate this approach, a case study of its application in the context of the Spanish Observatory for University Employability and Employment system has been developed, where users (Spanish universities and administrators) can control their own dashboards to reach insights about the employability of their graduates. These dashboards have been automatically generated through a domain specific language, which provides the syntax for specifying the requirements of each user. The domain language fuels a template-based code generator, allowing the generation of the dashboards' source code. Applying domain engineering to the dashboards' domain improves the development and maintainability of these complex software products given the variety of requirements that users might have regarding their graphical interfaces.

\section{INTRODUCTION}

The concept of employability has increasingly gained relevance over the last decades. There is a reason: knowing which factors increase the possibility to obtain a job or to perform better in current job positions could be decisive to improve individual and collective life quality.

However, this concept is still far away from having a straightforward definition (Chadha \& Toner 2017). As the literature suggests, employability can be seen as a capability to gain employment or as a set of skills and knowledge required to perform effectively in the workplace, among other definitions (Confederation of British Industry 2009; Hillage \& Pollard 1998; Yorke 2006). This lack of consensus when defining employability makes the research in this field a complicated task, given the fact that the definition of its factors depends on the perspective used to evaluate it, as well as the socioeconomic context in which employability and employment studies are framed. For these reasons, nowadays research on employability asks for an exploratory approach, to build stronger theoretical foundations.

Researching on employability has many potential benefits, aiming not only at knowing the variables that affect the capability to gain employment and have a successful work career, but 
58

59

60

61

62

63

64

65

66

67

68

69

70

71

72

73

74

75

76

77

78

79

80

81

82

83

84

85

86

87

88

89

90

91

92

93

94

95

96

also to exploit this knowledge to help policymakers and institutions with their missions. This knowledge can contribute to the creation of greater policies, focusing on the detected factors to enhance people's chances to obtain better employment. Specifically, educational institutions like universities could benefit from this knowledge. These institutions play a vital role regarding the employability of individuals (García-Peñalvo 2016), as they are in charge of transmitting knowledge and a series of skills to their students. By promoting the most relevant skills and capabilities that affect employability, it could be possible to increase the alignment of education with the labor market.

However, generating knowledge in such a study field is not a trivial task. As it has been introduced, there could be several variables involved in the research of students' employment and employability, so it is necessary to collect significant data volumes to be able to reach valuable insights. In addition to data collection, performing data analysis (Albright et al. 2010) is required to be able to reach useful insights. It is worth noting that analyzing employability data to identify and understand its factors could become a cornerstone in decision-making processes within educational institutions.

Nevertheless, even after performing data analysis, identifying patterns and indicators derived from the analysis outcomes remains a complex challenge. That is why it is crucial to assist decision-makers with powerful tools that allow reaching insights about the domain of the problem, to support decisions with complete and quality information (especially in the academic context, where these processes might have a series of social implications), that is, information and knowledge that has been extracted through visual analysis.

Information dashboards are one of the most commonly used software products for visual data analysis and knowledge extraction (Few 2006; Sarikaya et al. 2018). In a domain like employability, these tools can support exploratory studies through a set of graphical and interactive resources, allowing users to envision data more understandably (Tufte \& GravesMorris 2014) and identify relevant relations, indicators or patterns among large sets of data. It is essential to bear in mind that information dashboards are not just a set of aesthetic graphs and visualizations; they have to effectively transmit information to answer the questions of the users regarding the target domain. Moreover, this is not a trivial job, because of two main reasons: data and users themselves.

On the one hand, users do not have a set of standard and static requirements; they could demand different features or design attributes given their specific goals or needs. On the other hand, data is continuously increasing and evolving nowadays, so it is foreseeable that new information requirements will arise in time. Returning to the employability subject, information requirements in this domain might change in many different ways as this concept could demand new kind of 
97 variables or larger amounts of data to explore emerging dimensions or to perform more in-depth 98 analyses.

For these reasons, information dashboards not only need to be useful concerning functionality but also be customizable to adapt to specific user requirements. Also, they should be flexible and scalable regarding its data sources and structures, making the development and maintenance of information dashboards even more complicated. Of course, these issues could be addressed by developing particular dashboards for each involved user to achieve every specific goal, but clearly, this solution would be time-consuming and would require a lot of resources during the development and maintenance phases. Also, scalability would be almost impossible, as new users or changes in the requirements would necessarily imply more resources.

There are, nevertheless, a series of strategies to deal with these challenges. Specifically, software engineering paradigms like software product lines (Clements \& Northrop 2002; Gomaa 2004; Pohl et al. 2005) provide powerful theoretical frameworks to address flexibility, scalability and customization in software products that share sets of features within a common domain. Through the analysis of commonalities and variability points in the product domain, it would be possible to reduce the development and maintenance effort of building tailor-made solutions. This paradigm is potentially applicable to dashboards since these software products could be factored into sets of configurable components with configurable features. This paper describes the application of the SPL methodology to the dashboards' domain through the study of their characteristics and the definition of a DSL to manage the product derivation automatically. The main focus of this research is to test the potential usefulness and feasibility of this approach to manage fine-grained features that can be scattered through different code assets, and consequently, to provide a base method for generating personalized dashboard solutions to fit concrete user requirements.

The remainder of this work is structured as follows. Section 2 (Background) discusses the background of the problem of generating customized dashboards as well as their application to the employment and employability domain. Section 3 (Context) presents the application context and the motivation behind this pilot framework to generate dashboards to support visual analysis on university employment and employability data (framed within the Spanish Observatory for University Employability and Employment studies). Section 4 (Materials and Methods) describes the techniques used for the development of an initial approach to a generative dashboard framework. Finally, Section 5 exhibits the outcomes of this research to conclude with the discussion of the developed SPL and the conclusions derived from these results.

\section{BACKGROUND}

135 The main idea behind software product lines (SPLs) is that the final products can be derived 136 from a set of configurable core assets, allowing their adaptation to fit specific requirements. 
137 These core assets are developed during the domain engineering phase, in which commonality

138

139

140

141

142

143

144

145

146

147

148

149

150

151

152

153

154

155

156

157

158

159

160

161

162

163

164

165

166

167

168

169

170

171

172

173

174

175

176

and variability of the target product domain are identified to build a common base of components. Core assets are developed with variability points in which specific functionalities could be injected to obtain new products. Functionalities in SPLs are seen as features; the combination of the defined features within the scope of the line (generally following a feature model (Kang et al. 1990) allow stakeholders to build personalized products by reusing and assembling software components.

The SPL paradigm has been applied to a variety of domains: Mobile applications (Marinho et al. 2010; Nascimento 2008; Quinton et al. 2011); Applications for visualizing population statistics (Freeman et al. 2008); Sensor data visualizations (Logre et al. 2014); Variable content documents (Gómez et al. 2014); or e-Learning systems (Ezzat Labib Awad 2017).

These practical applications have proved the benefits of this paradigm. However, features usually refer to the software's logic, deflecting attention to the presentation layer. The idea of generating customized dashboards can be seen as a specific case of graphical user interfaces (GUI) automatic generation within SPLs. User interfaces require additional work regarding their implementation; they not only need to be functional but also usable to allow users to complete their tasks efficiently and achieve their goals. That is why the design of user interfaces is present through the whole development process, being time- and resource-consuming job.

Automation regarding GUI generation in software product lines has already been faced in several works. Generally, there is a lack of usability on the generated products that can be addressed by manually designing every product GUI. But this approach is highly inefficient in the SPL paradigm context since all the development time saved could be lost by introducing a manual task (Hauptmann et al. 2010). Integration of the GUI design process and the SPL paradigm is required to leverage the benefits of the two approaches (Pleuss et al. 2010). There is, as Pleuss et al. (2012) pointed out, a dilemma between automation and usability (Pleuss et al. 2012a; Pleuss et al. 2012b). To address this challenge, they utilized Model-Based UI Development (MBUID) methods to separate the functionality and the appearance of the GUI (Pleuss et al. 2010).

On the other hand, Gabillon et al. (2015) demonstrated the possibility of creating adaptive user interfaces through the Dynamic SPL (DSPL) paradigm and MBUID models by developing a context-aware data visualization tool that can be adapted during runtime.

DSPLs provide a useful paradigm for adapting code at run-time, obtaining adaptive GUIs. Kramer et al. (2013) proposed document-oriented GUIs with run-time variations through XML documents (Kramer et al. 2013). This context-adaptable feature has also been achieved by Sboui et al. (2018), by developing a mobile application that is also runtime adaptable through MBUID models and reusable artifacts. In this particular case, the code generation is based on eXtensible

Peer) Comput. Sci. reviewing PDF | (CS-2018:11:32512:2:0:NEW 7 May 2019) 
177 Stylesheet Language Transformations (XSLT) and XML files (Sboui et al. 2018). These works

178 shows not only the viability of GUI generation in the SPL/DSPL paradigms context but also their

179 valuable benefits.

180

181

It seems evident that GUI customization requires fine-grained features to achieve the desired

182 usability and design attributes. Fine-grained features mostly require annotative approaches regarding their implementation, given their specialization. Annotative approaches can address this issue because annotations can be arbitrarily specified at different source code fragments (Kästner \& Apel 2008; Kästner et al. 2008), and provide a framework for fine-grained automated software composition through feature structure trees (Apel et al. 2009).

187

188

189

190

191

192

193

194

195

196

197

198

199

200

201

202

203

204

205

206

207

208

209

210

211

212

213

214

215

216

There are different approaches to manage the implementation of variability at a fine-grained level (Gacek \& Anastasopoules 2001). Especially, frame- and template-based approaches provide valuable solutions to address this fine-grained level of variability, allowing the injection of particular fragments of code at any point of the base source code. Frame-based languages, like XML-based Variant Configuration Language (XVCL) (Jarzabek et al. 2003), provide a syntax to combine and insert fragments of code through the definition of frames, allowing the separation of concerns regarding the SPL implementation (Zhang et al. 2001). Templating can also achieve valuable results; templating libraries such as Jinja2 (Ronacher 2008) provide powerful functionalities to annotate the source code independently of the target programming language (Clark 2018; Ridge et al. 2017).

The generation of GUI within the context of a product family is still a convoluted field, although the previous work has enlightened the path to improve and leverage the automation and generation of these complex software elements. The complexity mainly comes from human factors and the vast variety of requirements regarding user interfaces.

This work aims to present an application of the SPL paradigm, in this case on the dashboards' domain, considering the fine-grained nature of their features and the necessity of customizing its interaction methods and visual appearance.

\section{CONTEXT}

The application of this work is framed within The Spanish Observatory for University Employment and Employability. The following subsections describe this organization's mission and the motivation to generate personalized dashboards to explore its data.

\section{The Observatory for University Employment and Employability}

The Observatory for University Employment and Employability (also known as OEEU, its Spanish acronym, http://oeeu.org) is an organization with the vision of becoming an information reference for understanding and exploiting knowledge about employment and employability of

Peer] Comput. Sci. reviewing PDF | (CS-2018:11:32512:2:0:NEW 7 May 2019) 
217 students from Spanish universities. To do so, this network of researchers and technicians conduct

218 studies about these fields in the academic context (Michavila et al. 2018a; Michavila et al. 2016;

219 Michavila et al. 2018b), through a data-driven approach to recollect, analyze, visualize and

220 disseminate employment and employability data of graduates from Spanish universities.

221

222

223

224

225

226

227

228

229

230

231

232

233

234

235

236

237

238

239

240

241

242

243

244

245

246

247

248

249

250

251

252

253

254

255

256

Firstly, in the data collection phase, universities provide their administrative records and, once this phase is completed, their students answer a questionnaire about different aspects of their education and work career. This process leaves the Observatory with a significant set of variables from the students' sample. For instance, in the 2015 study edition, more than 500 variables were gathered from 13006 bachelor students. Moreover, in the 2017 study edition, 376 variables were gathered from 6738 master degree students.

The volume of the data collected makes the presentation of the study results to the Observatory ecosystem's users a challenge, as the latter may have different requirements and necessities regarding the studies' data. For these reasons, an approach based on domain engineering fits the OEEU's needs, allowing an efficient generation of customized dashboards that meet different requirements.

\section{Motivation}

As it has been introduced, employment and employability are complex study fields that mainly ask for exploratory analysis, given its relatively initial status of research. In the context of the Spanish Observatory for University Employment and Employability, where a vast set of variables from significant quantities of students are recollected, it is crucial to rely on exploratory visualizations that allow users and administrators to identify at a glance unusual patterns or important data points by enhancing the understanding of the presented information (Card 1999).

In contrast with explanatory visualizations, in which the primary purpose is to tell a story through data, exploratory tools aim to facilitate users to pose more questions as data is being explored. In essence, explanatory analyses start from a question and use data to answer it. Exploratory analysis, on the other hand, uses data to detect new avenues of research. For instance, when a user does not have a clear question about the data, it will use an exploratory research to find patters or relations among variables. This same user could employ the acquired knowledge to explain the insights reached through previous explorations using an explanatory visualization.

Exploratory visualizations rely intensely on interaction to provide their functionality and to allow users to drill-down datasets, being able to discover new aspects of the domain by directly communicating with the graphical interface. However, an interaction can take many forms, and there is not a single solution to obtain usable and intuitive interfaces valid for every user.

Peer] Comput. Sci. reviewing PDF | (CS-2018:11:32512:2:0:NEW 7 May 2019) 
257

258

259

260

261

262

263

264

265

266

267

268

269

270

271

272

273

274

275

276

277

278

279

280

281

282

283

284

285

286

287

288

289

290

291

292

293

294

295

296

For instance, some users could find useful a visible control panel to manage data if they are going to apply filters, aggregations and so on intensively. On the other hand, other users can demand in-place interaction if they give more importance to having more space for the visualizations (instead of having a permanent control panel consuming screen space). Another example is that users that speak a left-to-right (LTR) or a right-to-left (RTL) language would demand different layouts for the same task, according to their sociodemographic or cultural context (Almakky et al. 2015; Marcus \& Gould 2000). Also, visualization novices could require task-oriented dashboards to support their visual analysis, since their past experience with this kind of tools is a relevant factor when interacting with a system (Elias \& Bezerianos 2011).

Once patterns, relations between variables and interesting dimensions have been identified through the exploration of data, even the exploratory nature of a dashboard can change for a more explanatory purpose to present the results understandably and strikingly.

For all these reasons dashboards, their components, their interaction, and even their primary purpose need advanced configuration and customization to fit into different contexts and requirements. Moreover, as it has been aforementioned, SPLs provide a potential solution to efficiently address this customization since visual components and interaction methods could be treated as features of the product line, decreasing the resources needed during the development and maintenance of dashboards.

\section{MATERIALS \& METHODS}

This section presents the materials and techniques used during the development of this first approach to a framework for generating dashboards to explore employment- and employabilityrelated variables.

\section{Meta-model}

The problem to address requires abstract modelling to capture basic features within the dashboards' domain. To do so, a meta-model is proposed. Meta-models are a crucial artefact in model-driven engineering and model-driven architectures (Kleppe et al. 2003), as they allow to define a high-level view of the domain without depending on specific technologies. Therefore, meta-models should remain as simple as possible to eventually, through a series of mappings and transformations, obtain concrete models (Álvarez et al. 2001).

For this generic dashboards' domain, the meta-model found in Fig. 1 is proposed. First of all, a specific user could handle a dashboard. This dashboard could be composed of one or more pages, being these last composed, in turn, by one or more containers. A container could be seen as a row or a column, and it can recursively contain more containers. The container recursion ends with a component, which is any graphic element that can be used in a dashboard. The 
297

298

299

300

301

302

303

304

305

306

307

308

309

310

311

312

313

314

315

316

317

318

319

320

321

322

323

324

325

326

327

328

329

330

331

332

333

334

335

336

recursion mentioned above allows the arrangement of any layout by the recurrent combination of rows and columns.

This meta-model eases the vision of the dashboards' domain, and it also allows to identify the common base of any dashboard.

\section{Feature model}

The meta-model gives a high-level vision of the dashboards' domain. However, it does not capture concrete features. That is why software product lines rely on feature models (Kang et al. 1990) to identify common and variable assets.

Feature models not only serve as a documentation element but also as an important artifact within the development process. The implementation of the core assets and the materialization of variability points on the code must be guided by the previously defined feature model.

In this domain, the feature model will capture the dashboards' visualization components, as well as individual features and restrictions of each visualization. The hierarchical structure of the feature model allows to define high-level characteristics and refine them through the tree structure until reaching the lower-level features (i.e., fine-grained features). This structure makes the scalability of features easier, since adding new features involves the addition of new nodes to the feature tree uniquely.

For the Observatory's dashboards, three main configurable visual components (features) have been defined: a scatter diagram, a chord diagram and a heat map. These visualizations address the requirements of the Observatory's data but can be reused for other data domains. Also, it is possible to specify a global filter that affects the data of all components previously defined. These high-level features of the dashboards' product line are presented in Fig. 2.

A detailed view of the scatter diagram feature can be seen in Fig. 3. It has a set of subsequent features, either mandatory, optional or alternative. One mandatory feature is the base logic of the scatter diagram (i.e., the component layout construction and its primary logic). Another mandatory feature is the initial data that the diagram will be showing on different dimensions since it must be specified. Among the optional features, it is possible to determine whether a tooltip will show up when hovering on data points if a set of controls will support the data exploration, or the capacity to zoom or export the diagram. Also, a title for the visualization can be included.

For the sake of simplicity, some of the lower-level features have been omitted in Fig. 3. For instance, the bar and panel control features have subsequent features. The detailed features for a panel type control are shown in Fig. 4 to provide an example. A control panel will rely on its 
337

338

339

340

341

342

343

344

345

346

347

348

349

350

351

352

353

354

355

356

357

358

359

360

361

362

363

364

365

366

367

368

369

370

371

372

373

374

375

376

underlying logic, and it can count on different optional features, like data selectors to dynamically change the visualization's presented data; in case of the $\mathrm{X}$ and Y axes, these selectors could be located within the control panel space or in-place controls (i.e., situated near the scatter diagram axes). Other possible features involve having an overview that shows a detailed view of a data point when hovering, data filters, among others.

The feature diagram provides a high-level and organized overview of the SPL, improving the organization of the source code and development tasks.

\section{Domain-specific language}

There is, however, a necessity of connecting the previous models to the dashboards' source code to be generated (Voelter \& Visser 2011). A Domain-Specific Language (DSL) has been designed to accomplish this connection. This DSL is based on the identified domain's features, by structuring them with XML technology (Bray et al. 1997) and by validating the model restrictions with an XML schema (Fallside 2000). XML technology provides a readable and easy-to-parse manner for injecting functionalities or requirements in a system, fostering flexibility since these rules are not directly defined (or hard-coded) in the source code.

The following examples describe the DSL developed for this work. Following the meta-model, every dashboard will be composed by one or more pages, each page with its configuration (i.e., layout and components, as seen in Fig. 5), and each page component with its setting (given the feature model, as seen in Fig. 6).

Data resources of each visual component are represented by the XSD generic type "anyType", to decouple the data structure and format from the presentation, and also to open up the possibility of injecting dynamic data sources without affecting the DSL syntax.

In Figures 6 and 7 the resemblance of the XML schema structure with the feature model can be appreciated. The hierarchical nature of XML matches with the hierarchical structure of feature diagrams. This resemblance allows better traceability of the features involved in the product line, because the syntax of the DSL is obtained from the feature model, thus providing a computerunderstandable specification of the SPL, necessary to process the requirements and to automate the dashboard generation. In this current approach, the dashboard's feature model serves as documentation, but, as it will be discussed, it would be extremely valuable to create a programmatic link between this model and the DSL specification, in order to propagate and reflecting any feature model change automatically in the DSL, improving maintainability.

Finally, Fig. 8 shows how the layout of the dashboard is specified in terms of rows, columns and components (following, again, the meta-model previously presented). The DSL combines both 
377 the meta-model and feature model designs to obtain a specific syntax to configure all the aspects

378 regarding the generation of final products.

379

380 The whole schema for the DSL can be consulted at the following GitHub repository

381 https://github.com/AndVazquez/dashboard-spl-assets (Vázquez-Ingelmo 2018).

382

383

\section{Code generator}

384 To put together all the developed assets and concepts, a code generator has been developed to 385 manage the generation of functional dashboards. The generator interprets the DSL (i.e., XML 386 configuration files) and selects the appropriate template (i.e., core assets of the SPL) to configure

387

388

389

390

391

392

393

394

395

396

397

398

399

400

401

402

403

404

405

406

407

408

409

410

411

412

413

414

415

416 them by injecting the chosen features, obtaining the dashboards' final source code. The code templates and XML configuration files are managed by the developers following the elicited user requirements.

The inputs and outputs of the code generator can be seen in Fig. 9.

\section{Code templates}

The next challenge regarding the implementation of this SPL involves the choice of the techniques for materializing the product line's variability points. In this case, personalization is focused on the visual elements of the system's presentation layer, which require fine-grained variability (Kästner et al. 2008). Coarse-grained variability involves the addition and removal of full components, which is also useful for this approach (users may prefer a scatter diagram over a chord diagram to achieve their goals, removing the last from the dashboard). However, visual components themselves (referring to the elements that compose them) also require high variability to fit into different requirements, so fine-grained variability needs to be accomplished. There exist different approaches to implement fine-grained software composition, as in the case of FeatureHouse (Apel et al. 2009), which uses superimposition and feature structure trees (FSTs), however, not every method supports the currently required granularity, which involves even statement-level variability. Fine granularity often prohibits superimposition approaches (Apel et al. 2013).

The mechanism chosen to reach the desired feature granularity is based on template engines. Template engines allow to tag sections and parameterize units of source code to inject concrete values later and obtain complete source files. This mechanism accomplishes the necessity of materializing the variable features of a tangible product of the line.

Jinja2 (Ronacher 2008) was selected as the engine for developing the core assets of this SPL. This template engine allows the definition of custom tags, filters and even macros, being the last one of the essential features to organize the core assets. As described in (Kästner et al. 2008), fine-grained approaches can make the source code tedious to read and maintain. By declaring 
417 every variant feature on different macros to compose them subsequently, it is possible to achieve

418 high cohesion and loose coupling on the SPL feature implementation process, improving

419 reusability and source code organization by grouping the different functionalities by its parent

420 feature. There was no need to implement extensions of the Jinja2 implementation and

421 mechanisms, as its current syntax was sufficient for the annotative approach followed.

422

423 A diagram of the detailed workflow for generating the source code can be seen in Fig. 10. The

424 code templates for this case study can be also consulted at

425 https://github.com/AndVazquez/dashboard-spl-assets (Vázquez-Ingelmo 2018).

426

427 RESULTS

428

429

\section{Generated dashboards}

430

431

432

As it has been already introduced, the Observatory collects important datasets to research the

433

employability and employment of graduates from Spanish universities. Relying on a

434 customizable exploratory tool would increase the chances of discovering interesting patterns or

435

436 relations within these complex fields. The dashboards of this case study have a series of particular requirements due to the data domain and the specific characteristics of the Observatory studies. For instance, the developed data visualizations exploit different dimensions of the

437

438 Observatory's collected variables. Also, the generated Observatory's dashboards needed to be connected to the organization's GraphQL API (Facebook 2016) that allow users to retrieve data statistics on demand (Vázquez-Ingelmo et al. 2017; Vázquez-Ingelmo et al. 2018a; VázquezIngelmo et al. 2018b; Vázquez-Ingelmo et al. 2018c), decoupling the data resources from the visual components' logic.

442

In this section, the results derived from the application of the presented dashboard product line within the university employment and employability domain are described. By tuning SPL through particular configurations, it is possible to obtain tailored solutions for different requirements and tasks.

447

Configuration \#1. Comparison of different values is one of the most relevant tasks regarding the exploration of university employability and employment data. These comparisons could enlighten which factors affect employability and employment to a greater or lesser extent, leading to the possibility of conducting deeper analyses.

452

For example, by configuring a dashboard with two scatter diagrams side by side, it is possible to apply different filters to each one and observe how data patterns evolve (Fig. 11). Also, adding the global reference feature to both diagrams helps to make comparisons by adding a reference 456 line marking the unfiltered and disaggregated values. 
457

458

459

460

461

462

463

464

465

466

467

468

469

470

471

472

473

474

475

476

477

478

479

480

481

482

483

484

485

486

487

488

489

490

491

492

493

494

495

496

It is possible to appreciate, for example, that men graduates are more optimistic when commenting opinions about their future wages and the possibility of developing a working career in Spain (Michavila et al. 2018b). However, these diagrams also allow seeing at a glance that Arts and Humanities and Sciences graduates are more pessimistic about their future than their counterparts in other branches of knowledge, which are more clustered. For instance, only $40 \%$ of Sciences women graduates think that they could have a working career in Spain within five years.

This configuration enables the user to explore data through the combination of different aggregations, variables and filters.

Configuration \#2. The previous configuration, however, could be complex for some users by having to control two diagrams at the same time to align different factors. A single scatter diagram could be added to the dashboard to drill-down data. It is possible to add another dimension to the scatter diagram component by mapping numerical variables through the radius of the visualization's data points.

For instance, following the same example of the first configuration, the differences between male and females can be observed by a gender aggregation of the data. In this case, the population of each group is mapped through the radius of the points (Fig. 12).

However, to see how the branch of knowledge affects the value of these variables, similarly to the previous configuration, it is necessary to continuously filter data by every single branch (Fig. 13). This configuration is then not recommendable when continuous, and more complex comparisons (such as the one made in the previous scenario) are required.

If, on the other hand, data exploration is not continuously required by a user, the controls could be allocated within a top bar (Fig. 14) that can be hidden to give more space to the visualizations.

Configuration \#3. On the other hand, different pages focused on different data variables or data dimensions could be configured. This functionality allows freedom when arranging the content of the dashboards' pages to make it understandable for every particular user.

In the Observatory's case, a user might prefer having the dashboard screens organized by the study edition, being able to navigate through them thanks to a navigation bar (Fig. 15).

Or if preferred, it could be specified that each page will exploit a different set of data variables; for example, having a single tab to explore the students' competences (Fig. 16).

Peer] Comput. Sci. reviewing PDF | (CS-2018:11:32512:2:0:NEW 7 May 2019) 
497 Through this view it is possible to see a misalignment between the perceived level that the 498 graduates have about their skills and the perceived level of contribution of the studies regarding 499 the acquisition of that skills, and also between that possessed level and the perceived required 500 level in their job positions (Michavila et al. 2018b).

501

502 The previous dashboards are a quite tiny set of the available combinations that can be achieved 503 through the SPL configuration, but they should serve as an example to show the possibilities of 504 having a framework for generating personalized dashboards.

505

506

507

508

509

510

511

512

513

514

515

516

517

518

519

520

521

522

523

524

525

526

527

528

529

530

531

532

533

534

535

\section{Product metrics}

The metrics for the SPL are the following regarding its feature model:

- Feature model height: 9

- Features: 146

- Optional features: 106

The number of valid configurations has been omitted, given the recursion of the dashboards' composition (as highlighted in the dashboard meta-model), so infinite valid configurations can be generated.

Regarding the core-assets (i.e. the templates' source code), the following metrics have been calculated (El-Sharkawy et al. 2018):

- Lines of feature code (LoF): 2638 lines of feature code. This metric is the addition of every line of code affected by any Jinja2 directive (i.e. every annotated line of code). It is a size metric that gives a high-level view about the source code associated to the SPL features.

- Fraction of annotated lines of code (PLoF): $48.39 \%$. This is a variability density metric showing that the SPL's products have a $51.61 \%$ of common code (2814 lines of code are not annotated).

536

- Scattering of variation points: this metric counts the number of times that a feature appears in the code (i.e. appears in a Jinja2 condition directive). High scattering values decreases the readability of the code. By refactoring the code into macros that contain all code associated to a specific feature, the scattering is reduced.

Given the complex domain in which the product line has been applied (i.e. the dashboards' domain), the scattering of the variation points was one of the main concerns, as high scattering would make the code even more complex. That was the reason to arrange the feature code into macros as a solution to address the scattering of variability points. 
537 Development time improvement

538

539

The development of the presented SPL, including its conceptualization and design, took 82 days,

540 as illustrated through a simplified Gantt diagram in Fig. 17. The core assets development task

541 includes all the artifacts regarding the SPL (i.e. the DSL, the templates, etc.).

542

543 Before implementing this approach, a dashboard template with the same components and KPIs

544 was the solution to offer all the results held in the Observatory's study, so universities could

545 compare their individual results with the global, aggregated results. The development of the

546 mentioned dashboard template took 15 days. However, this static approach limited universities

547

548

549 to freely explore their data, as mentioned in other sections.

550

551

552

553

554

555

556

557

558

559

560

561

562

563

564

565

566

567

568

569

570

571

572

573

574

575

576

Five of the 50 universities were interviewed to capture their dashboard requirements and to estimate the elicitation process time consumption. However, this estimation should be considered as speculative given the variability of the complexity of the elicitation process, and specially, given the number of different universities (i.e. users) involved. Nevertheless, the requirement elicitation took one day for the interviewed universities.

Given the project's potential continuity, the dashboard implementation process would mainly consume time regarding requirements elicitation by using the presented SPL approach, decreasing the time spent on development processes. Without this approach, the information dashboards implemented for future Observatory's employability study editions would remain static and generalized for each involved user.

Building a personalized dashboard consume resources in terms of requirement elicitation and design, but also in terms of implementation or development. If the development phases are automated, then the main benefit is not only decreasing the development time of individual dashboards, but also, if necessary, devoting more time to the requirements identification and design phases, which, in the end, are the backbone of well-constructed dashboards. That is why, although significant time was consumed for the implementation of the dashboard SPL ( 82 days), it can be seen as an investment for the future, specifically in environments where significant quantities of user profiles are involved.

\section{DISCUSSION}

The application of domain engineering and the SPL paradigm to identify and factorize information dashboard functionalities has shown its usefulness to generate different dashboards with a set of common assets through the study of the dashboards' domain. The obtained results are fairly valuable, and open new paths for applying this approach to other data domains with new requirements.

Dashboards are complex software solutions that could be highly beneficial when adequately

Peer] Comput. Sci. reviewing PDF | (CS-2018:11:32512:2:0:NEW 7 May 2019) 
577 designed and tailored for specific users. These products can support decision-making processes, 578 assisting visual analysis by presenting information in an understandable manner. However, the 579 variety of profiles involved in these processes and their different definitions of "understandable" 580 makes the implementation of dashboards a time- and resource-consuming task, since a dashboard 581 configuration that is highly useful for one user could be pointless for the rest of them. What is

582

583

584

585

586

587

588

589

590

591

592

593

594

595

596

597

598

599

600

601

602

603

604

605

606

607

608

609

610

611

612

613

614

615

616

617 more, dashboards can be composed of several elements, from simple visualizations to different linked views, cross-filtering capabilities, interaction methods, handlers, etc., thus making the dashboards' domain a complex domain not only because of the different profiles of potential users, but because of the great quantity of feasible combinations of these "dashboard elements" to build a proper solution. In addition, these features can be very fine-grained; in user-centered systems, a slight modification on visualization types, interaction patterns, layouts, color palettes, etc. could be crucial regarding the final perceived usability of the product.

Relying on a framework to easily generate information dashboards would allow stakeholders to focus on the information requirements and their refinement to provide better results when seeking valuable insights on large datasets. Also, it opens up the possibility to automatically adapt the dashboards' configurations to match dynamic requirements based on the device used (Cruz-Benito et al. 2018b) or other factors.

The factorization of the dashboards' components into individual features allow fine-grained reusability and a set of customization options. This fine-grained customization enables the possibility of having highly functional and exploratory-centered visualizations as well as more basic visual components more centered on the explanation of insights through the addition or removal of low-level features. The achieved granularity provides a foundation to develop not only whole visualization components, but also new interaction methods and design features that can be easily interchangeable to fulfill particular sets of user requirements.

An annotative method of implementation was undertaken using macros to encapsulate individual functionalities. This method takes all the benefits from the annotative approach (fine-grained customization) and avoids its code verbosity and scalability issues by dividing the core assets into base templates and macros (Kästner et al. 2008). Although there were other possibilities to implement the variability points, such as superimposition approach (which did not fulfilled the requirements for performing this approach, as discussed in the Materials \& Methods section) like the FeatureHouse framework (Apel et al. 2009; Apel \& Lengauer 2008) or the XVCL (Jarzabek et al. 2003) mechanism (which fits the feature granularity requirements of this domain), the final decision of using a templating engine allowed the direct connection of the designed DSL with the final source code, providing a higher level language to specify the dashboards' features, as well as the possibility of organizing the variability points into macros to increase readability, traceability and maintainability by having all the code associated to a feature in the same source file. 
618 The chosen technology to implement the DSL was XML. The decision of implementing directly

619 the DSL in XML technology was made because of the hierarchical nature of XML, and its

620 resemblance to the hierarchical structure of the feature diagram, thus being the designed DSL a

621 computer-understandable "translation" of the feature model for the dashboard generator to

622 process. However, this language could be not as human-readable as other DSL solutions,

623 generating issues if a non-expert user wants to specify its dashboards requirements by himself.

624 Creating a friendly user interface to allow the dashboards' feature selection without involving

625 direct manipulation of the XML files can be a valuable solution to address these issues and ease

626 the product configuration process in the future.

627

628 Customization at functionality level has illustrated to be straightforward, as it is possible to

629 easily vary the behavior of the visual components through the DSL. Visual design attributes

630 customization, however, needs to be faced more deeply, as only the layout composition can be

631 specified in detail at the moment. The visual customization challenge cannot be overlooked since

632 dashboards not only have to provide valuable functionality; they should offer that functionality

633 through a pleasant and usable interface (Few 2006; Sarikaya et al. 2018).

634

635 On the other hand, this work has addressed customization focused on the presentation layer of

636 dashboards, but with the SPL paradigm, architectural design can also be customized in order to

637 provide different functional features regarding data processing, interoperability, storage,

638 performance, security, etc., achieving a complete customizable dashboard solution, not only

639 focusing on the visual presentation.

640

641 Regarding data acquisition, the developed tool was integrated with the Observatory's GraphQL

642 API to provide dynamic data exploration. The connection to this particular type of data source

643 involved the implementation of specific connectors to decouple the visualizations from the

644 particular source. The variability of data sources is another identified challenge to be addressed

645 through this approach, to support different data formats or data structures. Although counting on

646 a GraphQL API facilitated the data retrieval by the unification of data requests, it is essential to

647 enable the specification of other data retrieval methods.

648

649 Product metrics showed that significant feature code was needed to address high customizability 650 of the dashboards ( $48.39 \%$ of the source code was annotated). Also, arranging the feature code 651 into macros helped to increase features' traceability as well as to decrease the scattering of the 652 variability points throughout the code, making the code more readable and maintainable.

653

654 The approach can decrease the development time of individualized dashboards for each involved 655 university. As presented in the results section, the SPL not only offered space for development 656 time improvements, but also enabled the capacity of offering customized solutions, which was 657 previously regarded as unviable given the time constraints of the Observatory's project. 
658 Embracing the SPL paradigm can be seen as an investment for the future for projects with a

659

660

661

662

663

664

665

666

667

668

669

670

671

672

673

674

675

676

677

678

679

680

681

682

683

684

685

686

687

688

689

690

691

692

693

694

695

696

697

common domain and with continuity over time.

Finally, it is clear that interesting patterns can be discovered thanks to the application of this dashboard SPL on the employability and employment fields. The Observatory's data provide a great context to perform more advanced analyses to enlighten this complex domain.

Having powerful visualization tools allow reaching insights about patterns or factors to guide the execution of more complex analyses and make decisions about the actions to take or the future research directions, like developing machine learning (ML) models (García-Peñalvo et al. 2018). Regarding this last field, having visualization tools to explore the input data before training any ML model could help to build better and more accurate models through an appropriate feature selection phase guided by the previously reached insights (Hall 1999).

The main weaknesses and limitations of this solution come from the preliminary nature of the framework; it is crucial to further validate the usability of the automatically generated products to show their usefulness to the main beneficiaries of the dashboards: the users, as well as assess its implementation in other domains. The approach needs to be further generalized to provide a more versatile method and to match also development requirements (available technology or preferred programming languages), although results seem promising. Automating the generation of dashboards given their goal, their context, their end users, etc. could be extremely beneficial due to the vast potential of impact that these tools have (Sarikaya et al. 2018).

\section{CONCLUSIONS}

A domain engineering approach has been applied to the dashboards' domain to obtain a SPL of this type of software solution. By the identification of commonalities and variability points, a dashboard meta-model has been developed as well as a feature model to capture the different customization dimensions of the SPL.

The SPL has been developed through an annotative approach using code templates and macros (forming the core assets of the family of products). A DSL has been designed to facilitate and automate the application engineering process. The configuration files based on the DSL feed a code generator in charge of adding or removing the product features. The presented approach was applied within the Spanish Observatory for University Employability and Employment system, to provide a variety of dashboard configurations that enable the exploitation and exploration of different dimensions regarding employability and employment data.

Future research lines will involve refinements of the meta-model and the DSL, usability testing of the obtained products and A/B testing (Cruz-Benito et al. 2018a; Cruz-Benito et al. 2018b; Kakas 2017; Siroker \& Koomen 2013) on different configurations. Architectural customization could be supported to add more coarse-grained features like a visualization recommendation

Peer] Comput. Sci. reviewing PDF | (CS-2018:11:32512:2:0:NEW 7 May 2019) 
698

699

700

701

702

703

704

705

706

707

708

709

710

711

712

713

714

715

716

717

718

719

720

721

722

723

724

725

726

727

728

729

730

731

732

733

734

735

736

737

738

739

740

engine (Gotz \& Wen 2009; Vartak et al. 2017; Voigt et al. 2012), interface language translation or data preprocessing techniques before its presentation. Finally, the customization levels of the dashboards' visual design and data sources need to be further addressed.

\section{REFERENCES}

Albright SC, Winston W, and Zappe C. 2010. Data analysis and decision making: Cengage Learning.

Almakky H, Sahandi R, and Taylor J. 2015. The Effect of Culture on User Interface Design of Social Media - A Case Study on Preferences of Saudi Arabians on the Arabic User Interface of Facebook. World Academy of Science, Engineering

Technology International Journal of Social, Behavioral, Educational, Economic, Business Industrial Engineering 9:107-111.

Álvarez JM, Evans A, and Sammut P. 2001. Mapping between levels in the metamodel architecture. International Conference on the Unified Modeling Language: Springer. $\mathrm{p}$ 34-46.

Apel S, Kastner C, and Lengauer C. 2009. FEATUREHOUSE: Language-independent, automated software composition. Proceedings of the 31st International Conference on Software Engineering: IEEE Computer Society. p 221-231.

Apel S, Kästner C, and Lengauer C. 2013. Language-independent and automated software composition: The FeatureHouse experience. IEEE Transactions on Software Engineering 39:63-79.

Apel S, and Lengauer C. 2008. Superimposition: A language-independent approach to software composition. International Conference on Software Composition: Springer. p 20-35.

Bray T, Paoli J, Sperberg-McQueen CM, Maler E, and Yergeau F. 1997. Extensible markup language (XML). World Wide Web Journal 2:27-66.

Card M. 1999. Readings in information visualization: using vision to think: Morgan Kaufmann.

Chadha D, and Toner J. 2017. Focusing in on employability: using content analysis to explore the employability discourse in UK and USA universities. International Journal of Educational Technology in Higher Education 14:33.

Clark S. 2018. Render your first network configuration template using Python and Jinja2. Available at https://blogs.cisco.com/developer/network-configuration-template.

Clements P, and Northrop L. 2002. Software product lines: Addison-Wesley.

Confederation of British Industry. 2009. Future fit: Preparing graduates for the world of work. CBI London.

Cruz-Benito J, Sánchez-Prieto JC, Vázquez-Ingelmo A, Therón R, García-Peñalvo FJ, and Martín-González M. 2018a. How different versions of layout and complexity of web forms affect users after they start it? A pilot experience. In: Rocha Á, Adeli H, Reis LP, and Costanzo S, eds. Trends and Advances in Information Systems and Technologies. Cham: Springer, 971-979.

Cruz-Benito J, Vázquez-Ingelmo A, Sánchez-Prieto JC, Therón R, García-Peñalvo FJ, and Martín-González M. 2018b. Enabling adaptability in web forms based on user characteristics detection through A/B testing and machine learning. IEEE Access 6:22512265. 10.1109/ACCESS.2017.2782678 
741

742

743

744

745

746

747

748

749

750

751

752

753

754

755

756

757

758

759

760

761

762

763

764

765

766

767

768

769

770

771

772

773

774

775

776

777

778

779

780

781

782

783

784

785
El-Sharkawy S, Yamagishi-Eichler N, and Schmid K. 2018. Metrics for analyzing variability and its implementation in software product lines: A systematic literature review. Information Software Technology.

Elias M, and Bezerianos A. 2011. Exploration views: understanding dashboard creation and customization for visualization novices. IFIP Conference on Human-Computer Interaction: Springer. p 274-291.

Ezzat Labib Awad A. 2017. Enforcing Customization in e-Learning Systems: an ontology and product line-based approach.

Facebook. 2016. GraphQL. Available at https://facebook.github.io/graphql/ (accessed 24th Jul. 2017.

Fallside DC. 2000. XML schema part 0: Primer. W3C, April 2000.

Few S. 2006. Information dashboard design.

Freeman G, Batory D, and Lavender G. 2008. Lifting transformational models of product lines: A case study. International Conference on Theory and Practice of Model Transformations: Springer. p 16-30.

Gacek C, and Anastasopoules M. 2001. Implementing product line variabilities. ACM SIGSOFT Software Engineering Notes: ACM. p 109-117.

García-Peñalvo FJ. 2016. The Third Mission. Education in the Knowledge Society 17:7-18.

García-Peñalvo FJ, Cruz-Benito J, Martín-González M, Vázquez-Ingelmo A, Sánchez-Prieto JC, and Therón R. 2018. Proposing a machine learning approach to analyze and predict employment and its factors. International Journal of Interactive Multimedia and Artificial Intelligence 5(2):39-45. 10.9781/ijimai.2018.02.002

Gomaa H. 2004. Designing Software Product Lines with UML: From Use Cases to PatternBased Software Architectures: Addison Wesley Longman Publishing Co., Inc.

Gómez A, Penadés MC, Canós JH, Borges MR, and Llavador M. 2014. A framework for variable content document generation with multiple actors. Information and Software Technology 56:1101-1121.

Gotz D, and Wen Z. 2009. Behavior-driven visualization recommendation. Proceedings of the 14th international conference on Intelligent user interfaces: ACM. p 315-324.

Hall MA. 1999. Correlation-based feature selection for machine learning.

Hauptmann B, Bauer B, Harhurin A, and Pleuss A. 2010. Supporting derivation and customization of user interfaces in software product lines using the example of web applicationsMaster's thesis. Technische Universität München.

Hillage J, and Pollard E. 1998. Employability: developing a framework for policy analysis: DfEE London.

Jarzabek S, Bassett P, Zhang H, and Zhang W. 2003. XVCL: XML-based variant configuration language. Proceedings of the 25th International Conference on Software Engineering: IEEE Computer Society. p 810-811.

Kakas AC. 2017. A/B Testing.

Kang KC, Cohen SG, Hess JA, Novak WE, and Peterson AS. 1990. Feature-oriented domain analysis (FODA) feasibility study. Carnegie-Mellon Univ Pittsburgh Pa Software Engineering Inst.

Kästner C, and Apel S. 2008. Integrating compositional and annotative approaches for product line engineering. Proc GPCE Workshop on Modularization, Composition and Generative Techniques for Product Line Engineering. p 35-40.

Peer] Comput. Sci. reviewing PDF | (CS-2018:11:32512:2:0:NEW 7 May 2019) 
786

787

788

789

790

791

792

793

794

795

796

797

798

799

800

801

802

803

804

805

806

807

808

809

810

811

812

813

814

815

816

817

818

819

820

821

822

823

824

825

826

827

828

829

830

831
Kästner C, Apel S, and Kuhlemann M. 2008. Granularity in software product lines. Proceedings of the 30th international conference on Software engineering: ACM. p 311-320.

Kleppe AG, Warmer J, and Bast W. 2003. MDA Explained. The Model Driven Architecture: Practice and Promise. Addison-Wesley Longman Publishing Co., Inc., Boston, MA.

Kramer D, Oussena S, Komisarczuk P, and Clark T. 2013. Using document-oriented GUIs in dynamic software product lines. ACM SIGPLAN Notices: ACM. p 85-94.

Logre I, Mosser S, Collet P, and Riveill M. 2014. Sensor data visualisation: a composition-based approach to support domain variability. European Conference on Modelling Foundations and Applications: Springer. p 101-116.

Marcus A, and Gould EW. 2000. Crosscurrents: cultural dimensions and global Web userinterface design. interactions 7:32-46.

Marinho FG, Lima F, Ferreira Filho JB, Rocha L, Maia ME, de Aguiar SB, Dantas VL, Viana W, Andrade RM, and Teixeira E. 2010. A software product line for the mobile and context-aware applications domain. International Conference on Software Product Lines: Springer. p 346-360.

Michavila F, Martínez JM, Martín-González M, García-Peñalvo FJ, and Cruz Benito J. 2018 a. Empleabilidad de los titulados universitarios en España. Proyecto OEEU. Education in the Knowledge Society 19:21-39. 10.14201/eks20181912139

Michavila F, Martínez JM, Martín-González M, García-Peñalvo FJ, and Cruz-Benito J. 2016. Barómetro de empleabilidad y empleo de los universitarios en España, 2015 (Primer informe de resultados). Madrid: Observatorio de Empleabilidad y Empleo Universitarios.

Michavila F, Martínez JM, Martín-González M, García-Peñalvo FJ, Cruz-Benito J, and VázquezIngelmo A. 2018b. Barómetro de empleabilidad y empleo universitarios. Edición Máster 2017. Madrid, España: Observatorio de Empleabilidad y Empleo Universitarios.

Nascimento LMd. 2008. Cores assets development in software product lines-towards a practical approach for the mobile game domain.

Pleuss A, Botterweck G, and Dhungana D. 2010. Integrating automated product derivation and individual user interface design.

Pleuss A, Hauptmann B, Dhungana D, and Botterweck G. 2012a. User interface engineering for software product lines: the dilemma between automation and usability. Proceedings of the 4th ACM SIGCHI symposium on Engineering interactive computing systems: ACM. p 25-34.

Pleuss A, Hauptmann B, Keunecke M, and Botterweck G. 2012b. A case study on variability in user interfaces. Proceedings of the 16th International Software Product Line ConferenceVolume 1: ACM. p 6-10.

Pohl K, Böckle Gn, and Linden FJvd. 2005. Software Product Line Engineering: Foundations, Principles and Techniques: Springer-Verlag New York, Inc.

Quinton C, Mosser S, Parra C, and Duchien L. 2011. Using multiple feature models to design applications for mobile phones. Proceedings of the 15th International Software Product Line Conference, Volume 2: ACM. p 23.

Ridge B, Gaspar T, and Ude A. 2017. Rapid state machine assembly for modular robot control using meta-scripting, templating and code generation. Humanoid Robotics (Humanoids), 2017 IEEE-RAS 17th International Conference on: IEEE. p 661-668.

Ronacher A. 2008. Jinja2 Documentation. Welcome to Jinja2-Jinja2 Documentation (2.8-dev). Sarikaya A, Correll M, Bartram L, Tory M, and Fisher D. 2018. What Do We Talk About When We Talk About Dashboards? IEEE Transactions on Visualization Computer Graphics. 
832 Sboui T, Ayed MB, and Alimi AM. 2018. A UI-DSPL Approach for the Development of

833

834

835

836

837

838

839

840

841

842

843

844

845

846

847

848

849

850

851

852

853

854

855

856

857

858

859

860

861

862

863

864

865

866

867

868

869

870 Context-Adaptable User Interfaces. IEEE Access 6:7066-7081.

Siroker D, and Koomen P. 2013. A/B testing: The most powerful way to turn clicks into customers: John Wiley \& Sons.

Tufte E, and Graves-Morris P. 2014. The visual display of quantitative information.; 1983.

Vartak M, Huang S, Siddiqui T, Madden S, and Parameswaran AJASR. 2017. Towards visualization recommendation systems. 45:34-39.

Vázquez-Ingelmo A. 2018. Code repository that supports the research presented in the paper "Taking advantage of the software product line paradigm to generate customized user interfaces for decision-making processes: a case study on university employability". Available at https://github.com/AndVazquez/dashboard-spl-assets.

Vázquez-Ingelmo A, Cruz-Benito J, and García-Peñalvo FJ. 2017. Improving the OEEU's datadriven technological ecosystem's interoperability with GraphQL. In: Dodero JM, Ibarra Sáiz MS, and Ruiz Rube I, eds. Fifth International Conference on Technological Ecosystems for Enhancing Multiculturality (TEEM'17) (Cádiz, Spain, October 18-20, 2017) New York, NY, USA: ACM.

Vázquez-Ingelmo A, García-Peñalvo FJ, and Therón R. 2018a. Application of domain engineering to generate customized information dashboards. HCI International 2018 20th Conference on Human-Computer Interaction (15-20 July 2018). Las Vegas, Nevada, USA: Springer.

Vázquez-Ingelmo A, García-Peñalvo FJ, and Therón R. 2018b. Domain engineering for generating dashboards to analyze employment and employability in the academic context. 6th International Conference on Technological Ecosystems for Enhancing Multiculturality. Salamanca, Spain.

Vázquez-Ingelmo A, García-Peñalvo FJ, and Therón R. 2018c. Generation of customized dashboards through software product line paradigms to analyse university employment and employability data. Learning Analytics Summer Institute Spain 2018 - LASI-SPAIN 2018. León, Spain.

Voelter M, and Visser E. 2011. Product line engineering using domain-specific languages. Software Product Line Conference (SPLC), 2011 15th International: IEEE. p 70-79.

Voigt M, Pietschmann S, Grammel L, and Meißner K. 2012. Context-aware recommendation of visualization components. The Fourth International Conference on Information, Process, and Knowledge Management (eKNOW): Citeseer. p 101-109.

Yorke M. 2006. Employability in higher education: what it is-what it is not: Higher Education Academy York.

Zhang H, Jarzabek S, and Swe SM. 2001. XVCL approach to separating concerns in product family assets. International Symposium on Generative and Component-Based Software Engineering: Springer. p 36-47. 
Figure 1

Dashboard meta-model

The dashboard meta-model allows a high level view of the target domain.

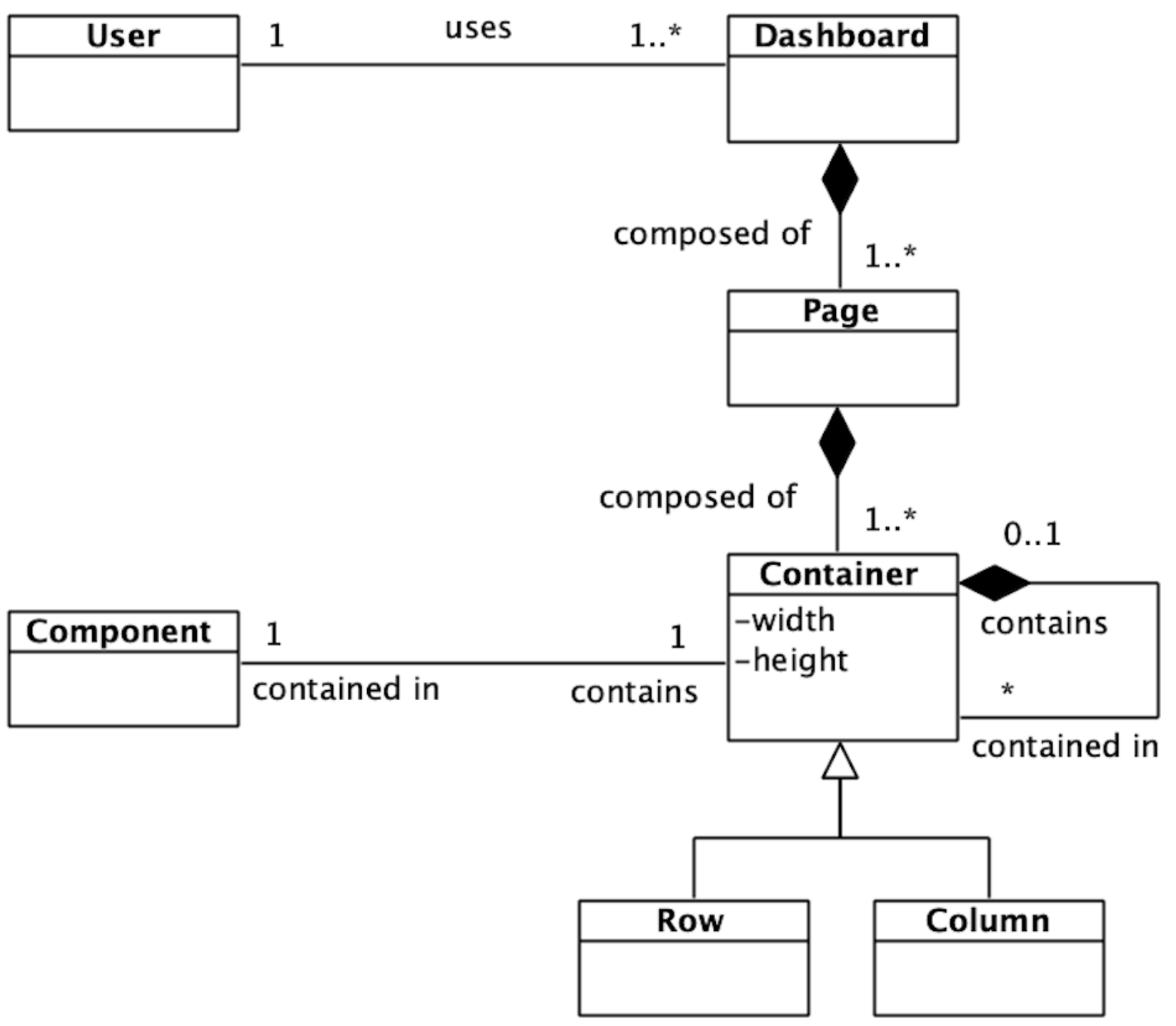


Figure 2

High-level view of the feature diagram

This feature diagram shows high-level components that could compose the dashboard.

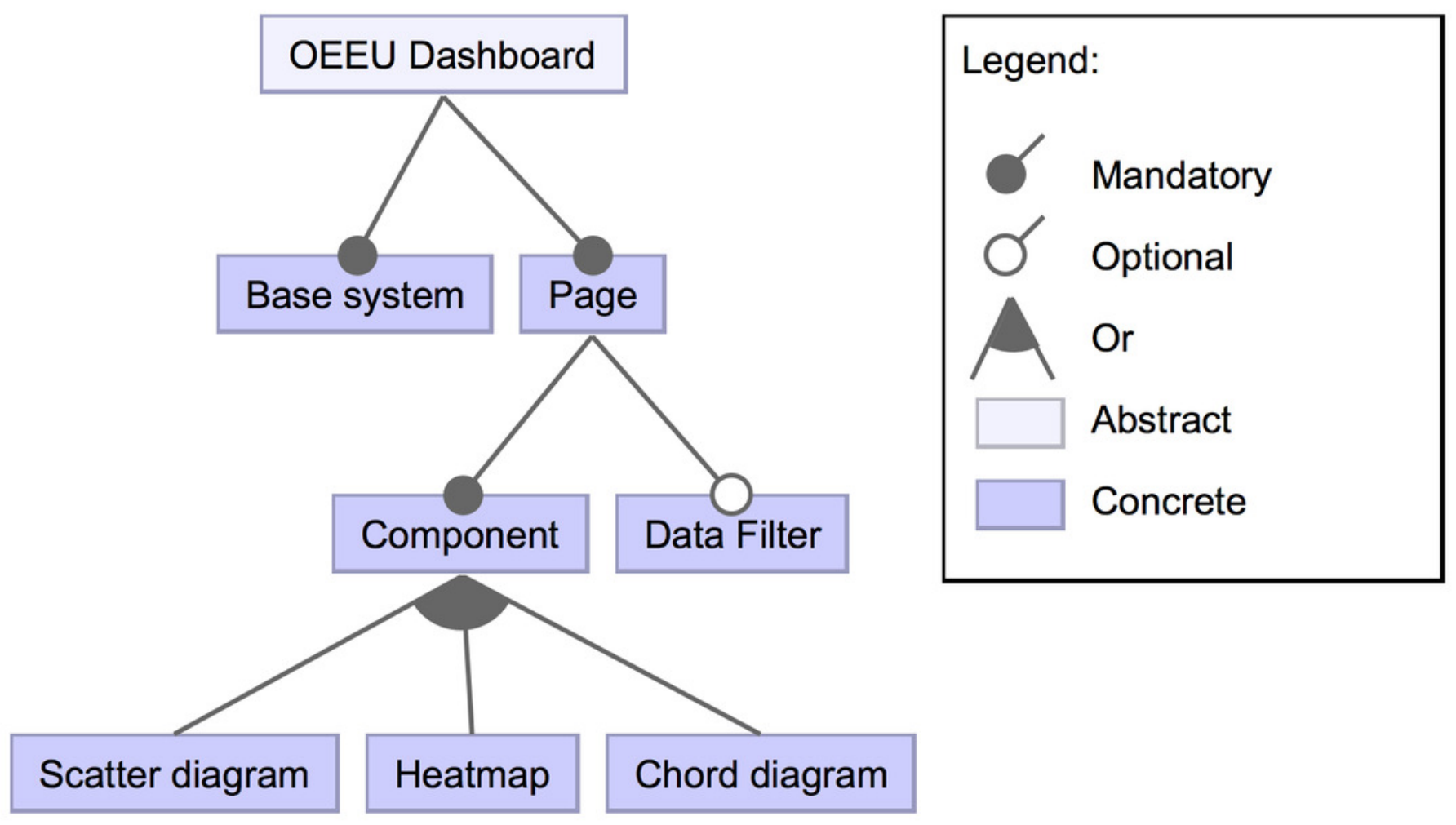


Figure 3

High-level view of the scatter diagram component's features

This snippet of the feature model shows the possible features regarding the scatter diagram component.

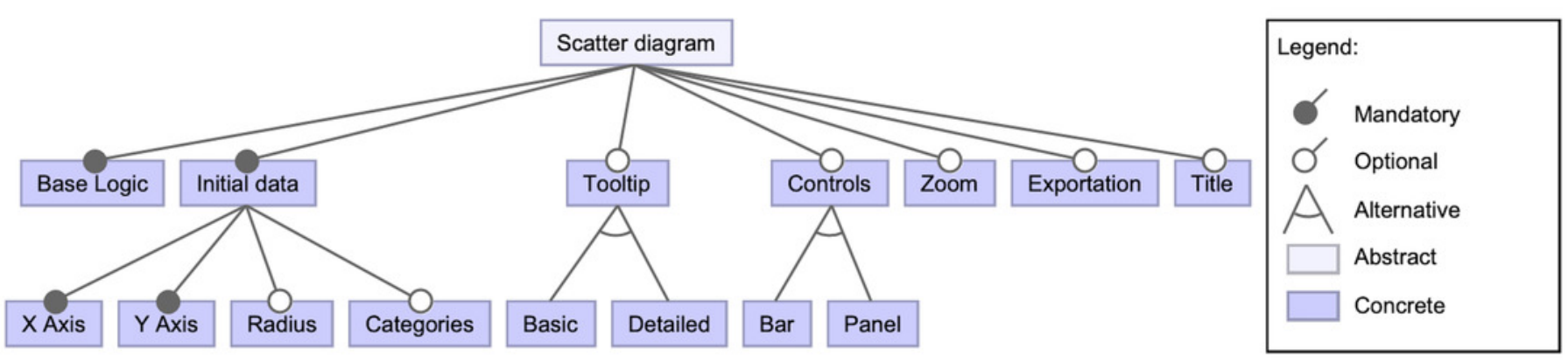


Figure 4

High-level view of a component's panel subsequent features

This part of the feature diagram shows lower-level features regarding the components' control panel.

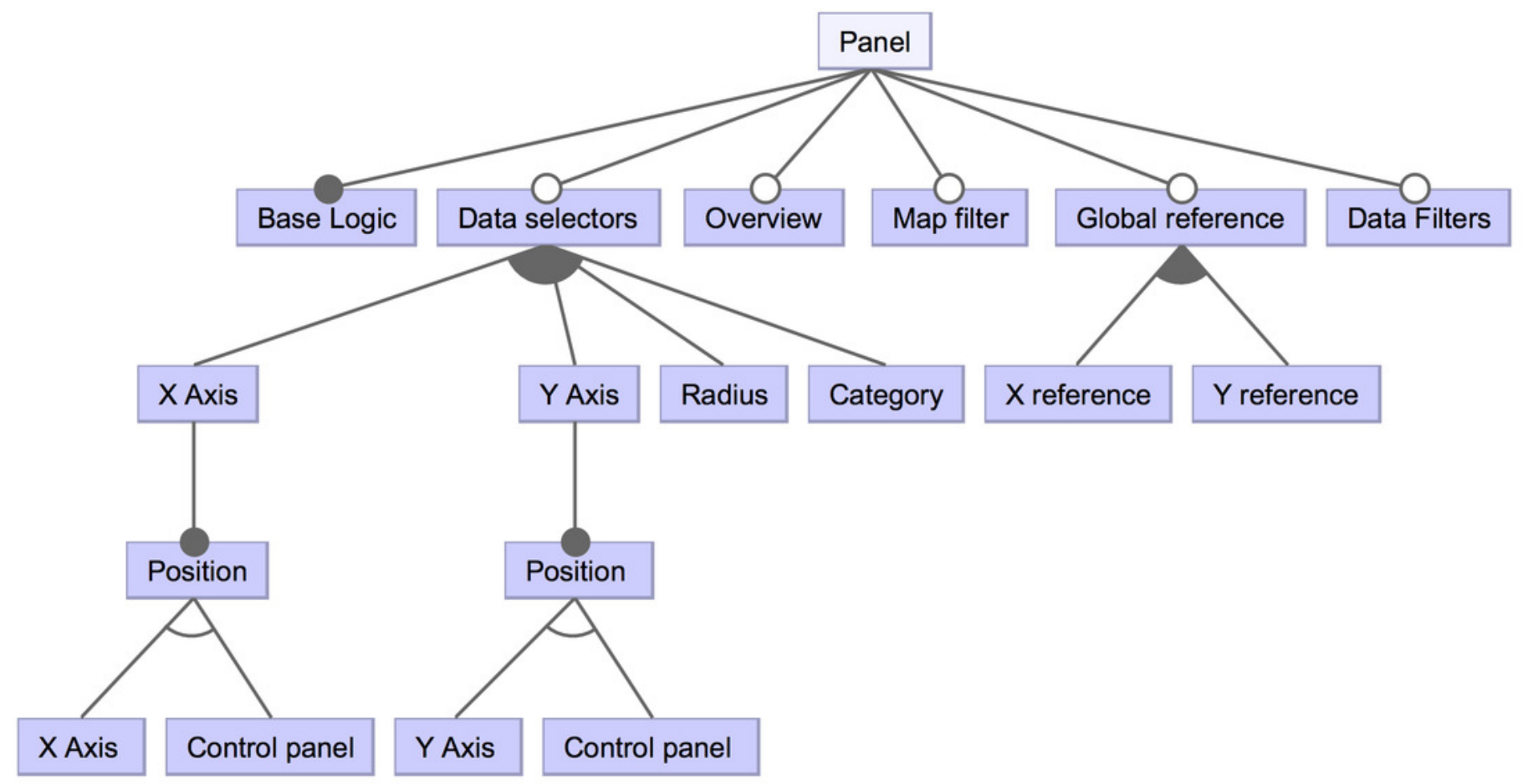




\section{Figure 5}

Snippet of the DSL schema

It is possible to specify the dashboard layout and its elements (i.e. data filters, components, etc.)

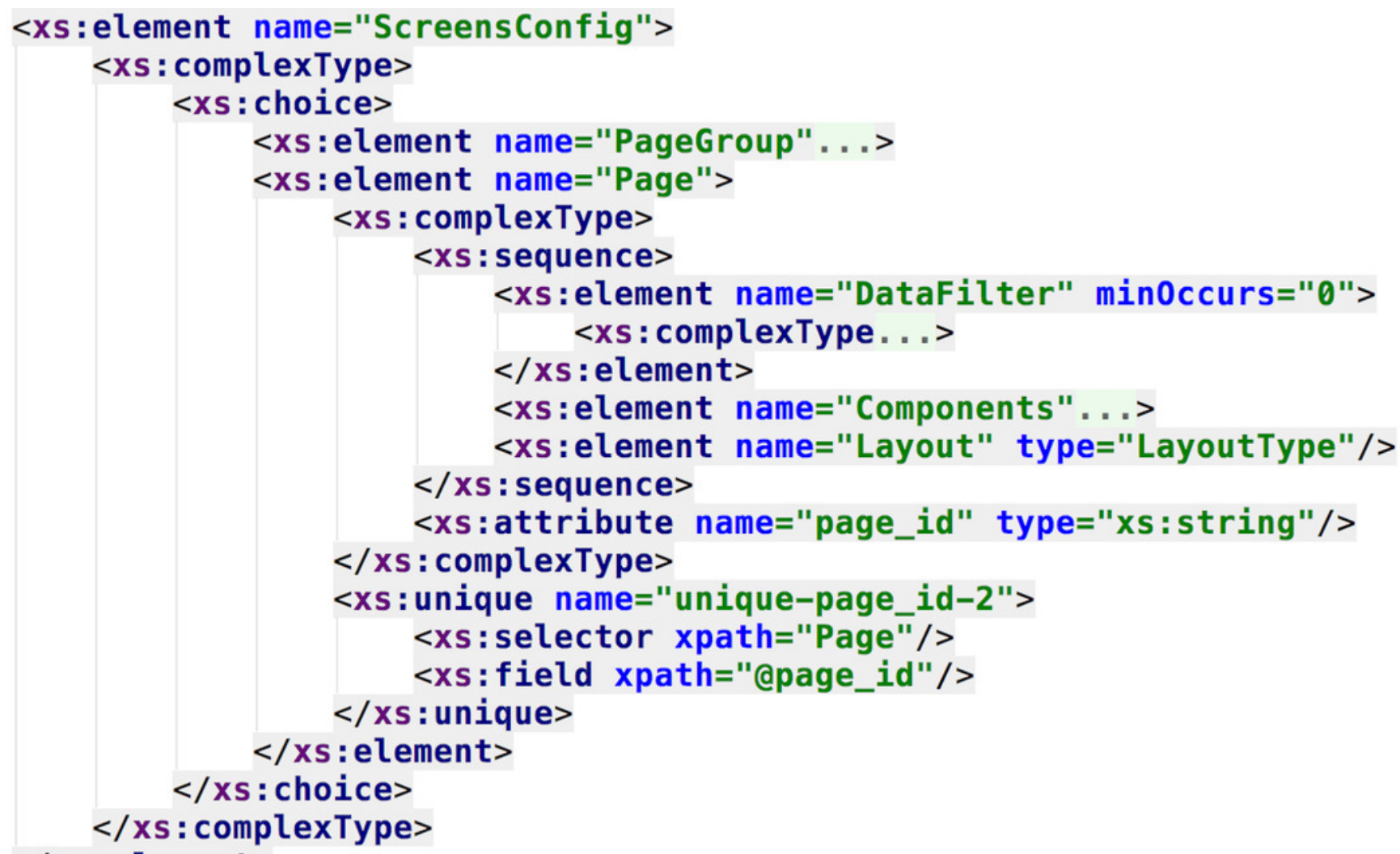

$</$ xs: element $>$ 


\section{Figure 6}

DSL schema regarding the specification of the dashboard components

It is possible to see the link between the feature model elements and the XML schema elements (e.g. the components that could compose the dashboard)

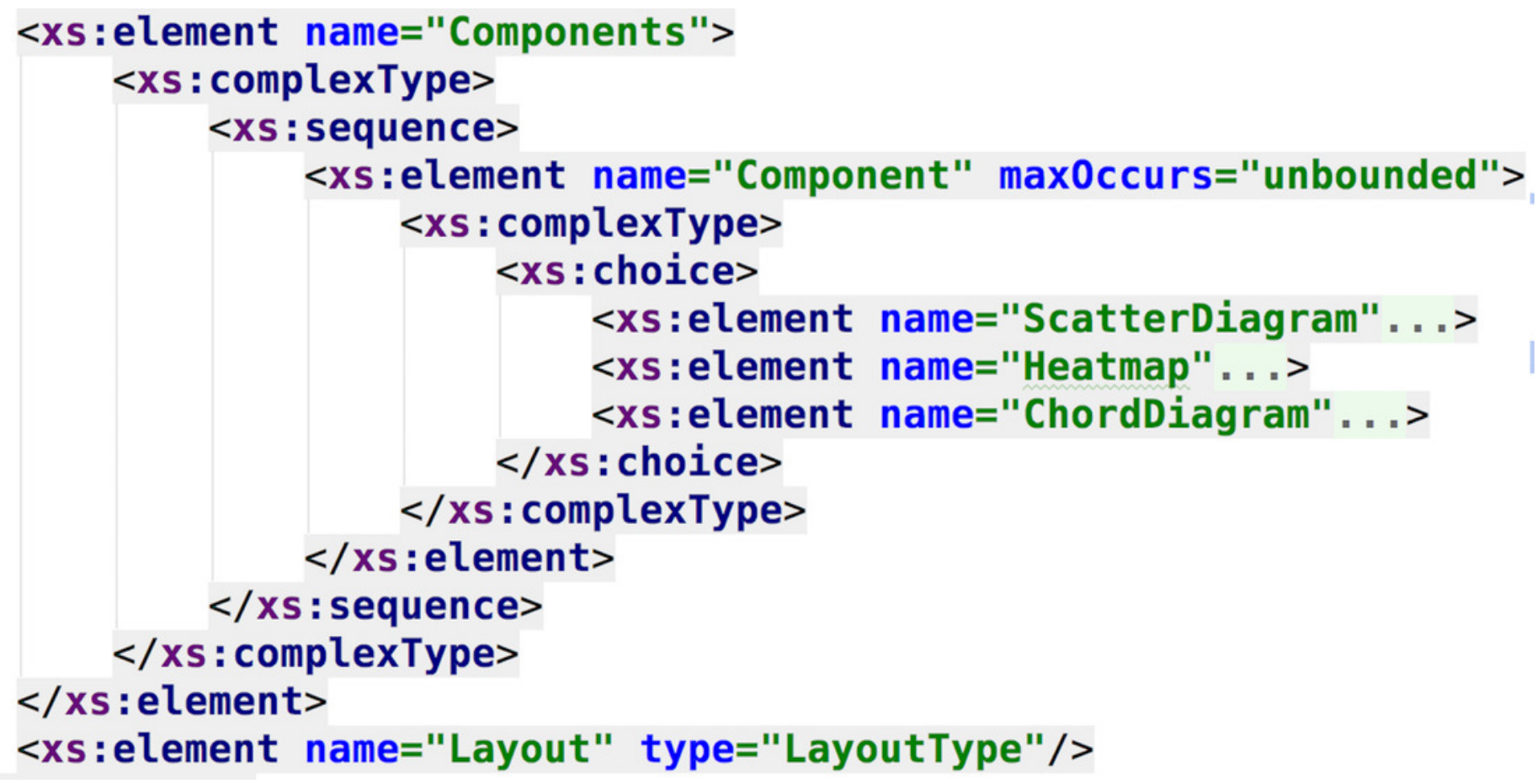


Figure 7

DSL schema regarding the specification of the scatter diagram component

This part of the DSL represents the available features for the scatter diagram component.

<xs: element name="ScatterDiagram">

$<x s$ : complexType>

$<x s$ : sequence>

<xs:element name="Title" type="xs:string"... >

$<x$ : element name="Zoom" type="xs:string"... >>

$<x$ : element name="Exportation"... >

$<x$ s:element name="InitialData"... >

$<x s$ : element name="Controls" minoccurs="0"... >

$<x s$ :element name="Tooltip" minoccurs="0"... >

$</ x s$ : sequence $>$

$<x s$ :attribute name="component_id"

type="xs: string"/>

$</ x s$ : complexType $>$

$</ x s$ : element $>$ 


\section{Figure 8}

XML type for specifying the dashboard's layout

The dashboard layout (previously modeled through the dashboard meta-model) is specified

by creating a custom type.

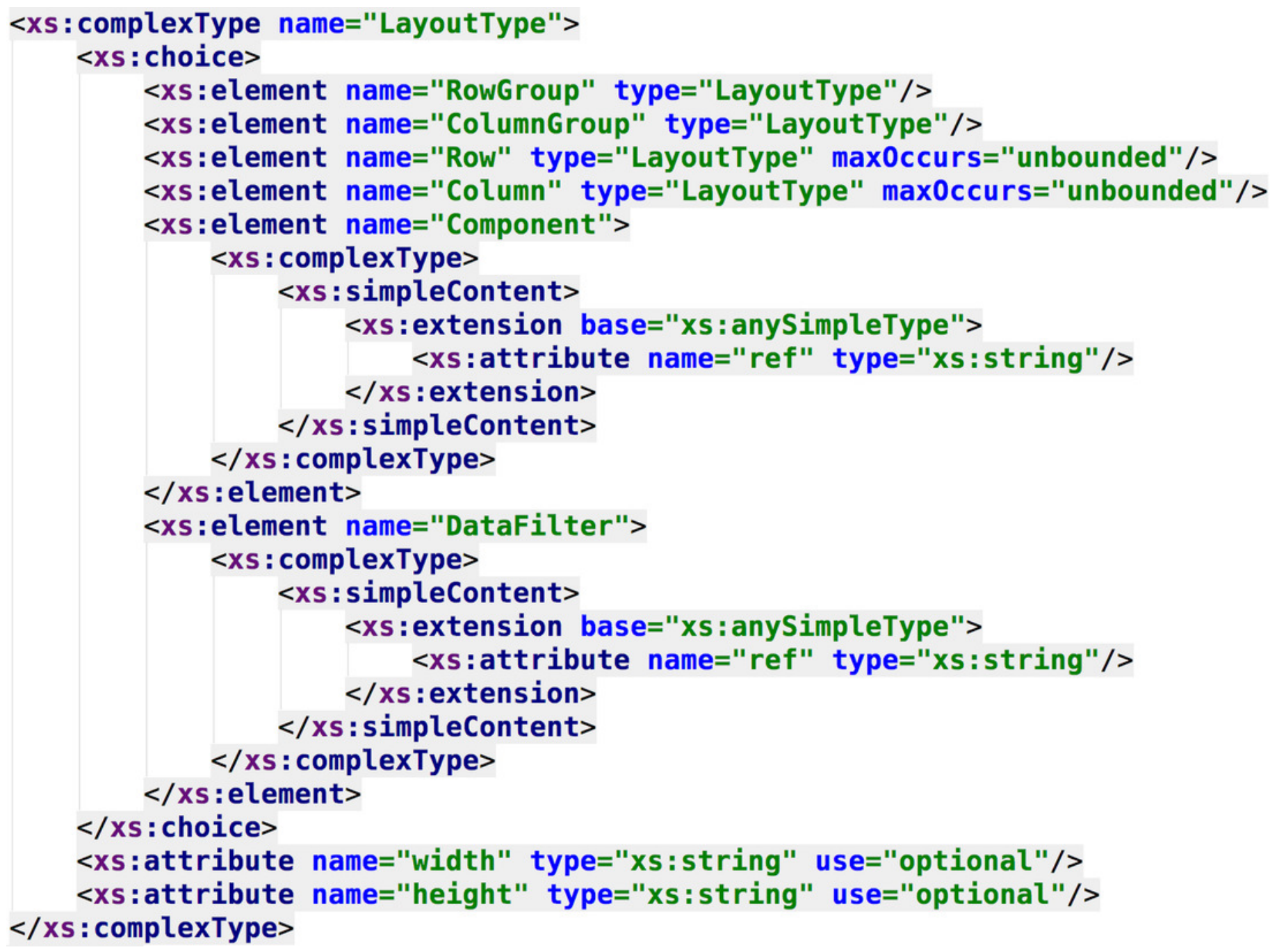


Figure 9

Code generator inputs and outputs

The code generator is fed with the code templates and the XML configuration files to provide the final source code of the dashboard.

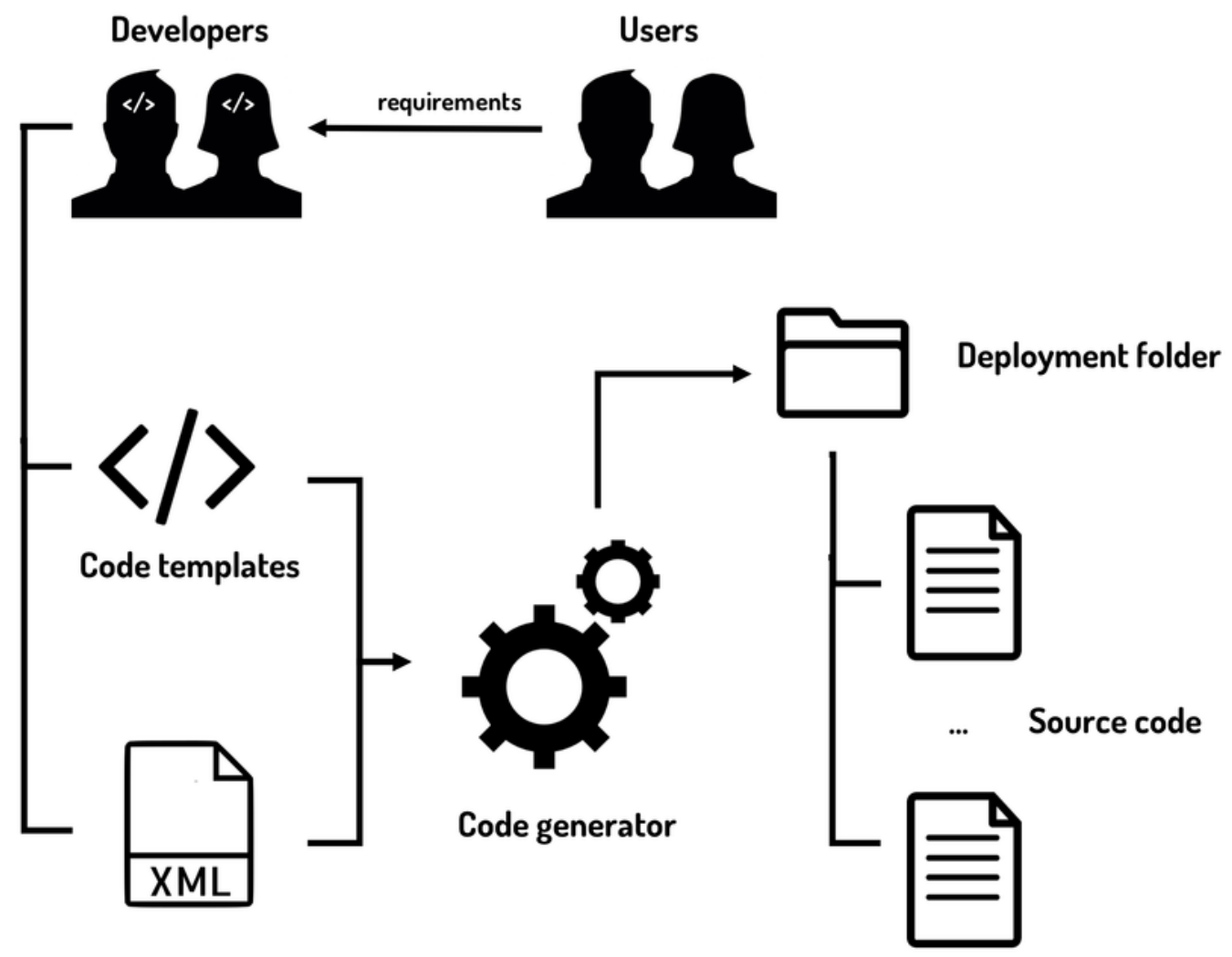

XML configuration 


\section{Figure 10}

\section{Workflow of the code generation process}

\section{A simplified view of the code generator behavior.}

\section{Template}

function my(selection) \{

selection. each (function () \{

var tooltipscatterDiagram $=\mathrm{d} 3$. select ("body") . append ("div")

.attr("class", "tooltip")

.attr("id", "compare-tooltip")

.style("display", "none")

.style("opacity", 0);

\footnotetext{
$\mathrm{xScale}=\mathbf{d} \mathbf{3}$. scaleLinear () -range( [0, width]);

$\mathrm{yScale}=\mathrm{d} \mathbf{3}$. scaleLinear() .range([height, 0]);

rScale $=\mathbf{d} 3 \mathbf{s}$ scaleLog( $)$ -range([10, radius]);
}

$\{\{$ chart_title. render_chart_title ()$\}\}$

$\{$ c control_bar. render_control_bar( () \}\}

$\{\{$ render_structure. render_component_structure () \}\}

$\{\{$ control_panel. render_control_panel('query_handler', 'vis_id') \}\}

$\{\{$ export_functionality.export() $\}\}$ _tooltip( 'vis_id' ) $\}\}$

$\{\{$ axis_functionality. render_axis_handlers ('xText', 'yText', 'vis_id') \}\}

\section{Generated source code}

.style("float", "left") .style("position", "relative")

.style("width", width + "px")

.attr("id", "vis_container_ScatterDiagram_1"

d3. select ("\#save-ScatterDiagram_1")

.on ("mouseover", function() \{

d3. select(this).style("cursor", "pointer");

\})

d3. select(this).style("opacity", 1);

.on ("mouseout", function() f

d3. select(this).style("cursor", "default"),

\})

d3. select(this).style("opacity", 0.3);

.on("click", function() \{

d3. select (this). style("opacity", 0);

saveSvgAsPng (d3.select ("\#original_svg_ScatterDiagram_1"). node( ), "ScatterDiagram_1" + '.png', 1 backgroundColor: 'white',

\})

$$
\text { scale: } 4
$$

\});

d3. select ("body")

. append ("div")

.attr("class", "tooltip")

.attr("id", "overview-tooltip-" + vis_id)

\section{Component configuration}

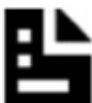

Macro

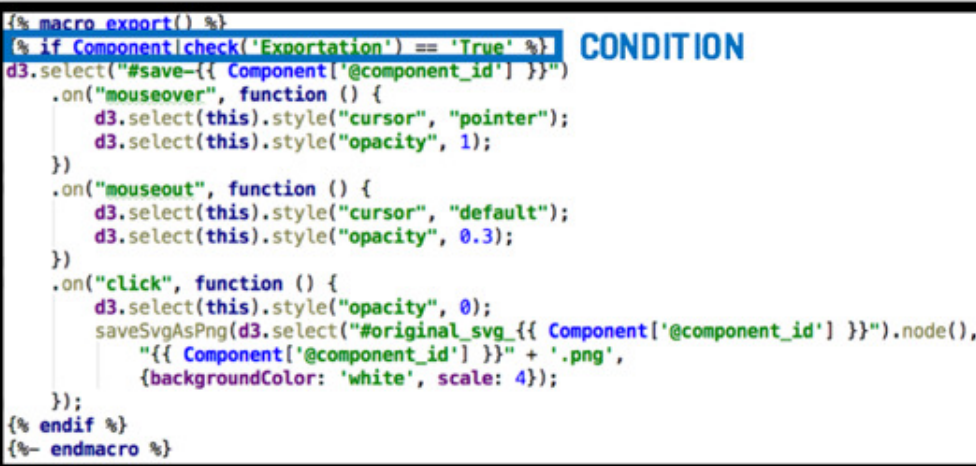


Figure 11

Results derived from the first configuration

Through this configuration is possible to apply different filters simultaneously to each scatter diagrams to observe how patterns evolve
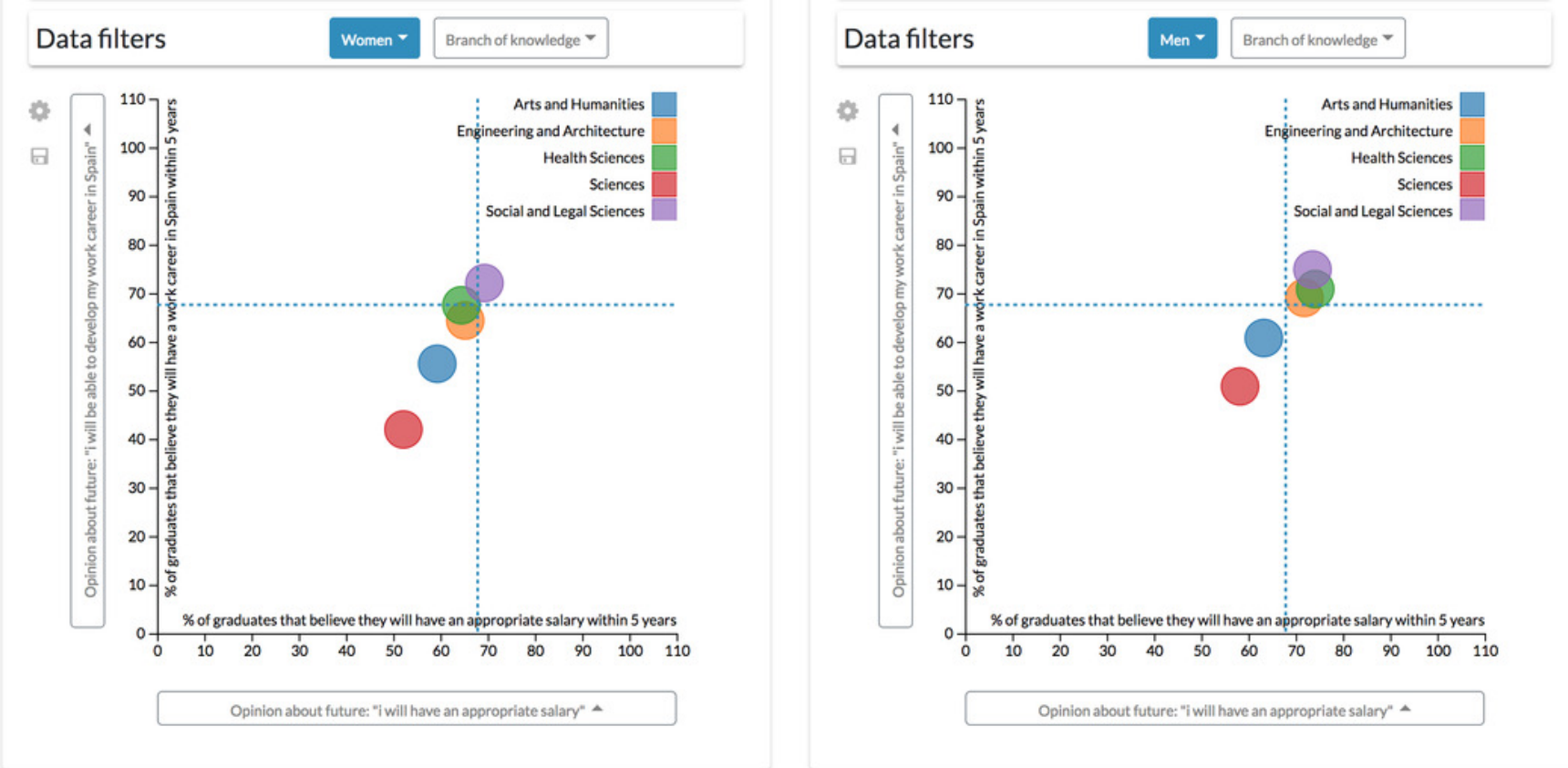
Figure 12

Results derived from the second configuration

The scatter diagram shows the link between different students' opinions classified by gender.

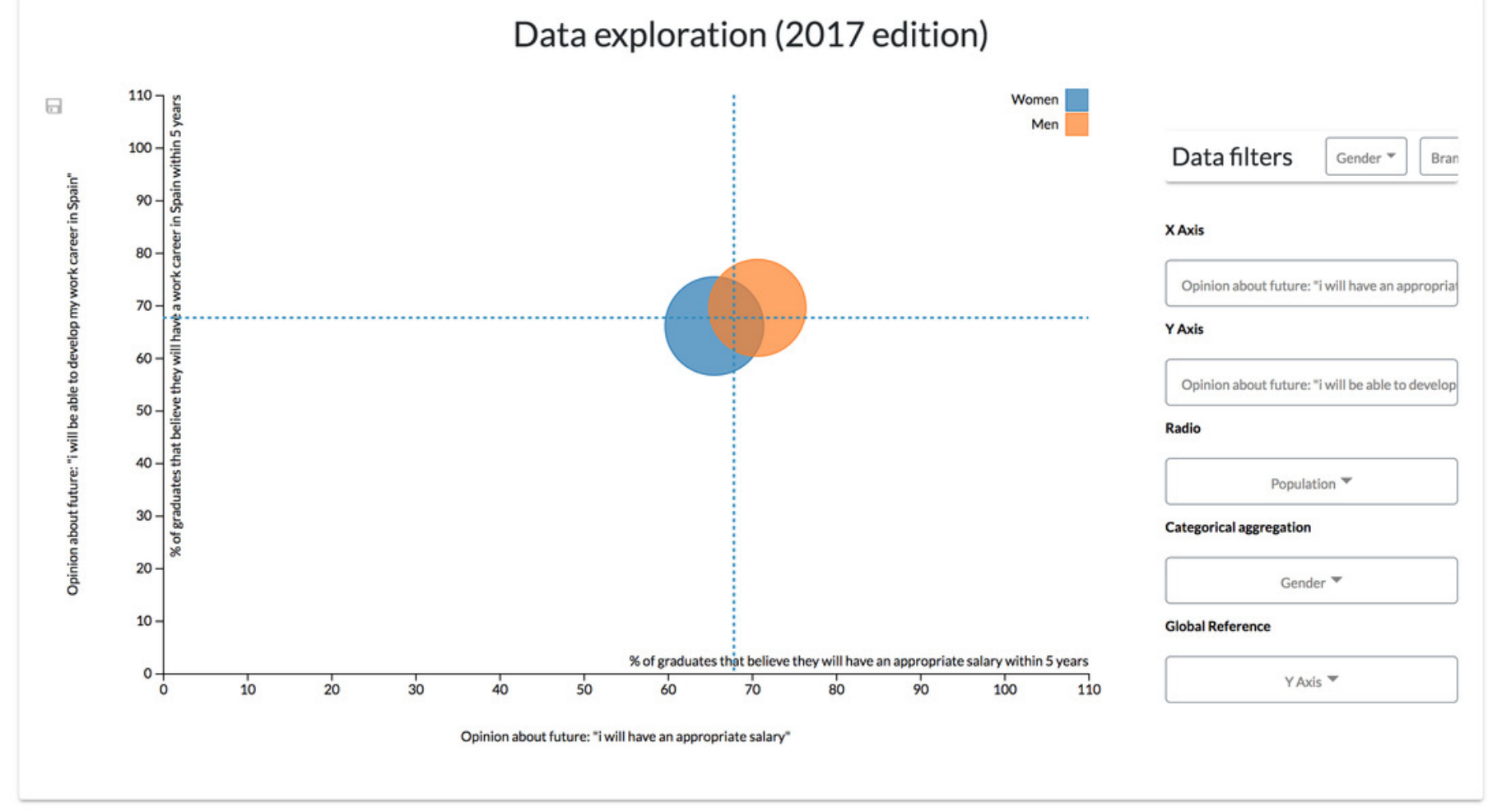


Figure 13

Results derived from the second configuration

The scatter diagram shows the link between different students' opinions classified by gender and filtered by the branch of knowledge, showing only the results related to Science students.

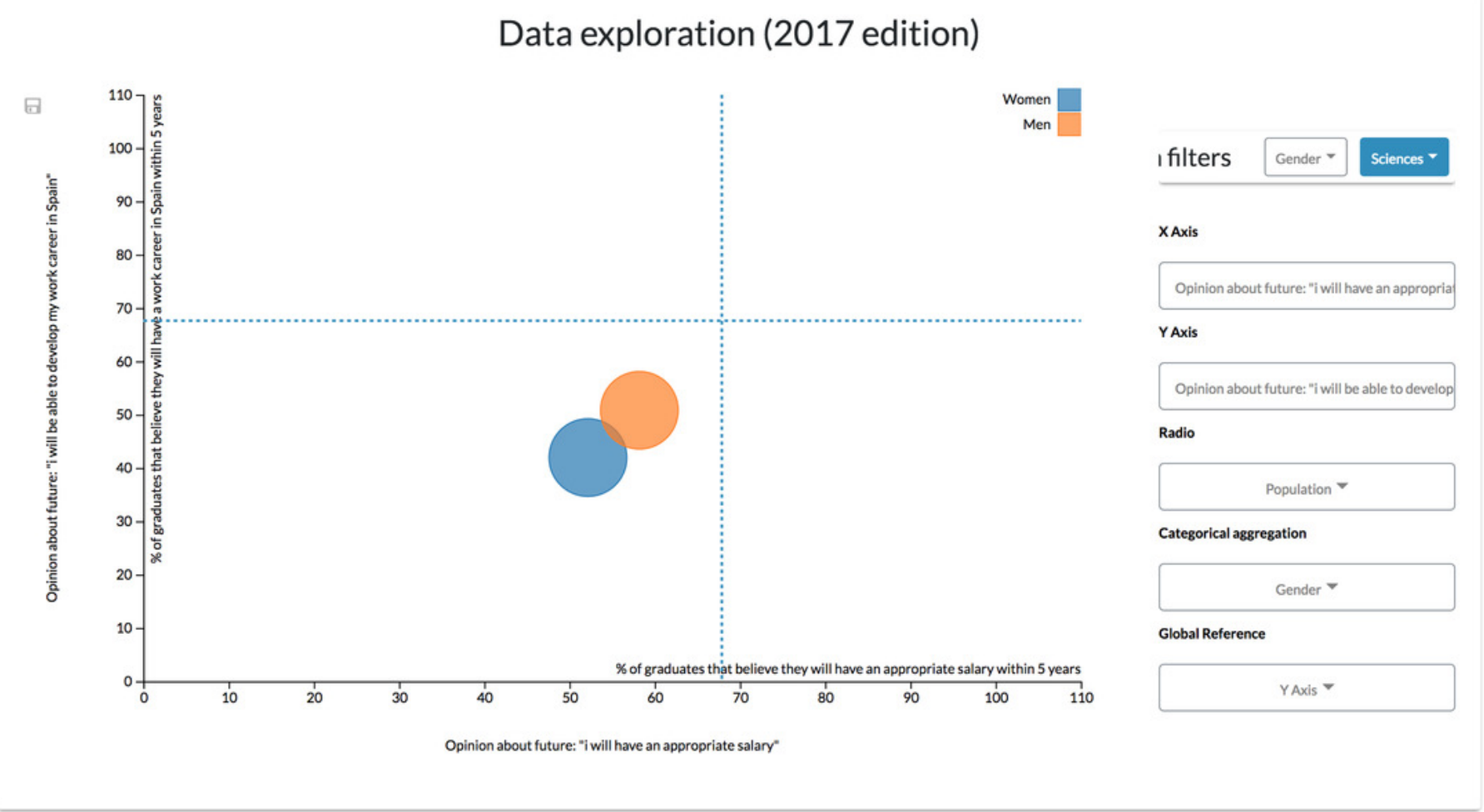


Figure 14

Modification of the second configuration to change the controls location

The controls for the scatter diagram are arranged in a bar on top of the visualization.

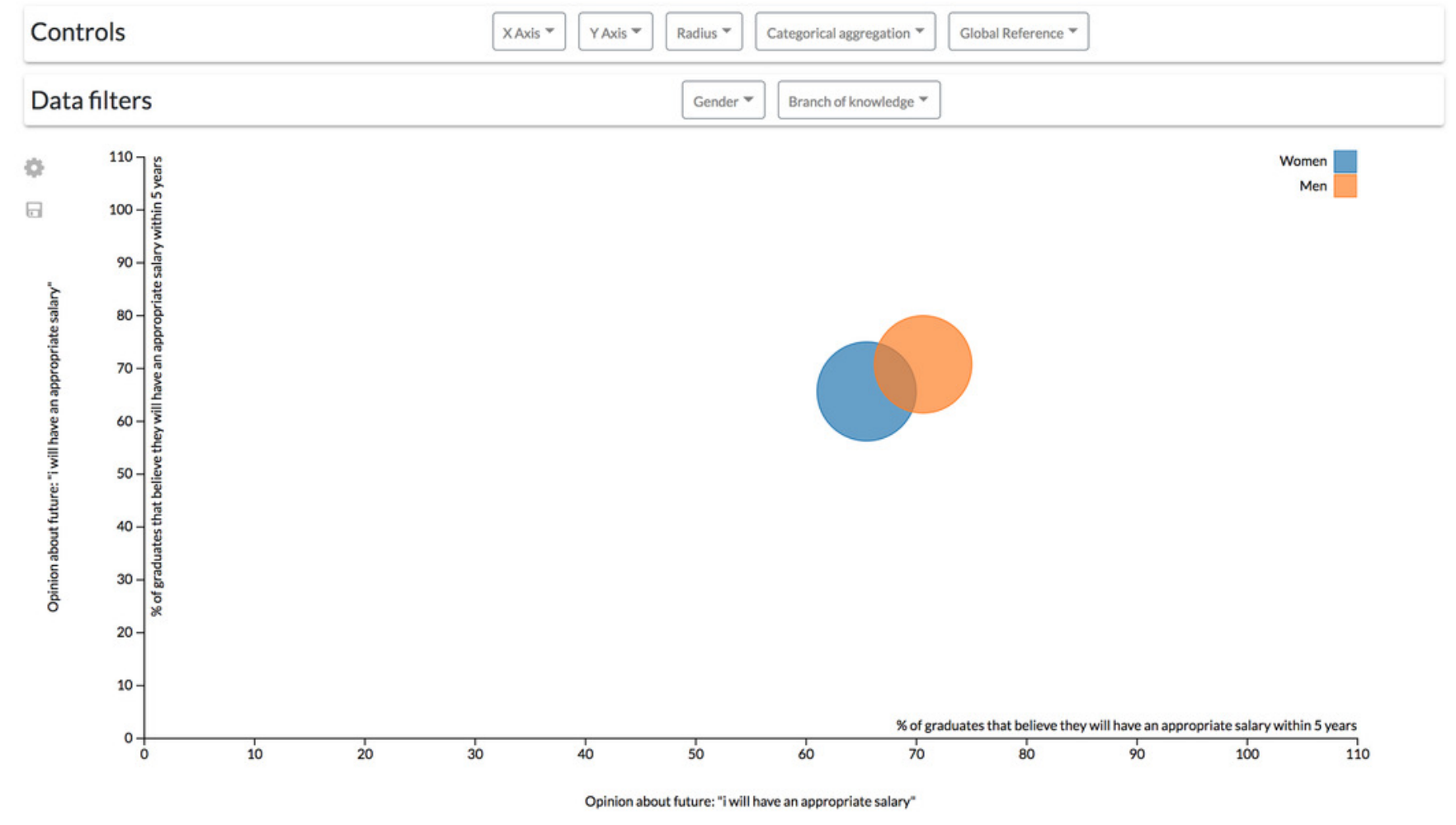




\section{Figure 15}

Dashboard involving different information visualizations

By specifying the layout of the dashboard it is possible to achieve dashboards with different components, each one with its own features. 


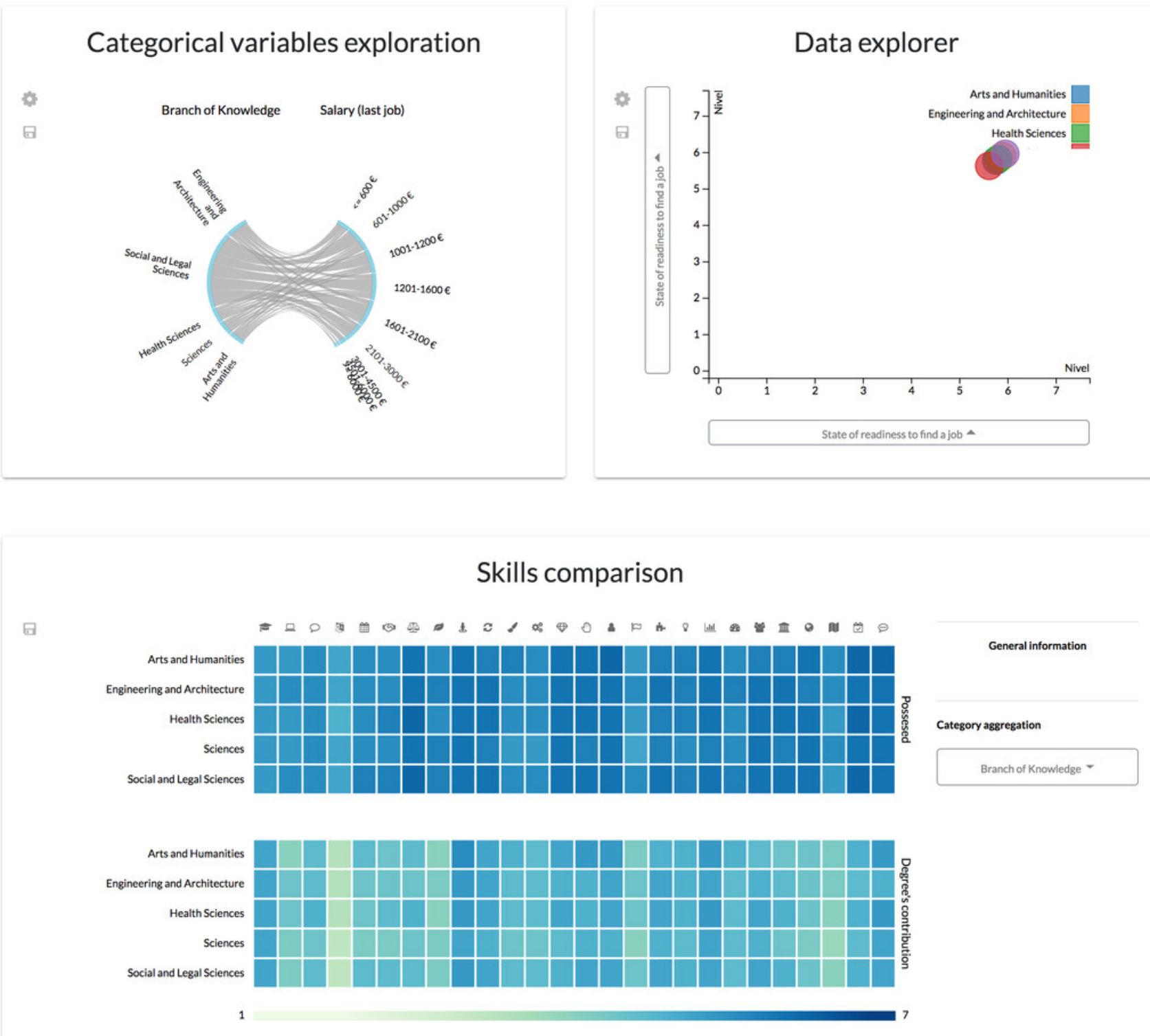




\section{Figure 16}

Possible layout configuration for comparing students' skills through different study editions

This configuration can be useful to identify lack of skills at-a-glance or their evolution through time. 
Graduates skills (2017)

क

回
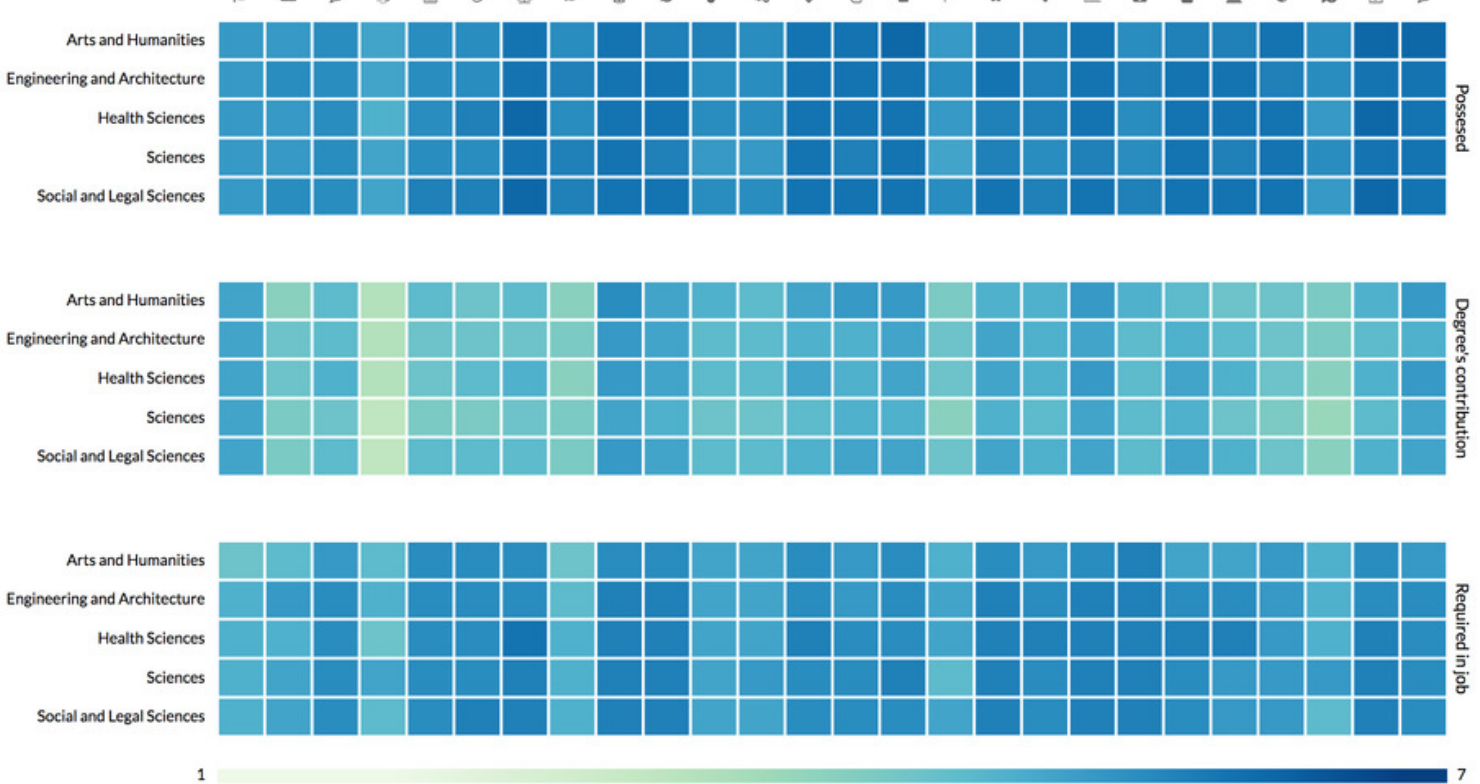

Graduates skills (2015)

\$

回
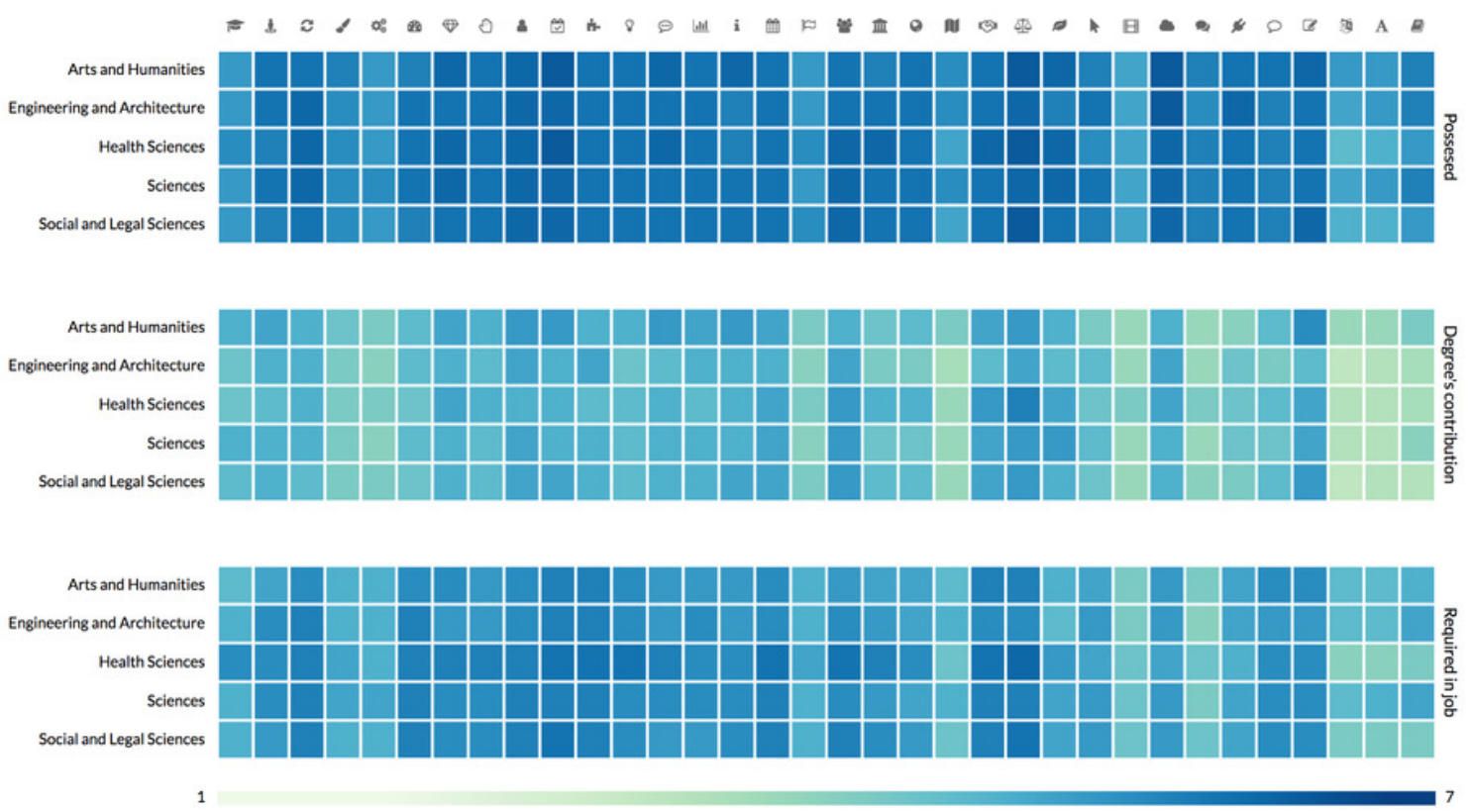


\section{Figure 17}

Simplified Gantt diagram of the SPL development

The Gantt diagram shows each task regarding the SPL development including its contextualization and design.

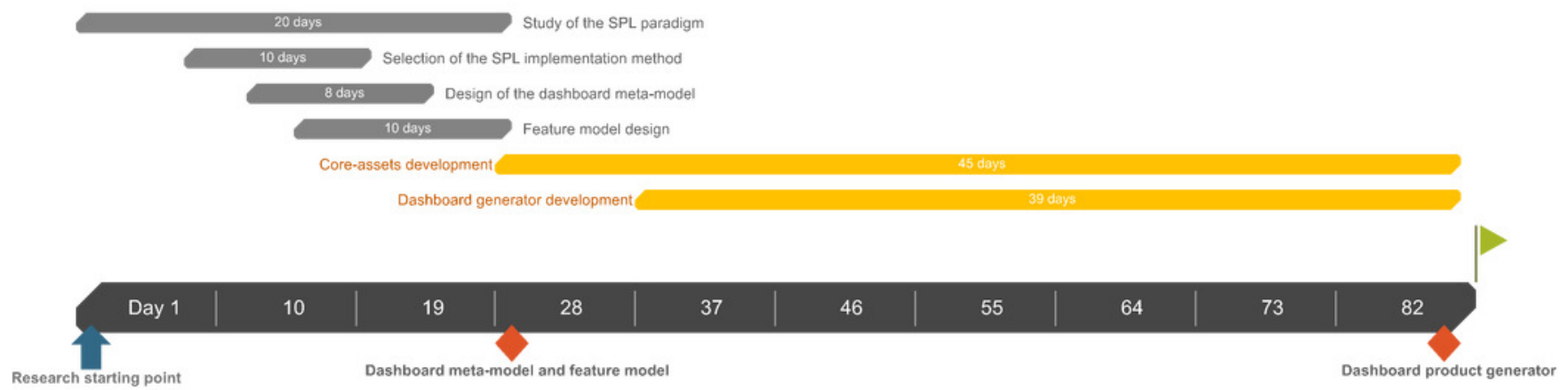

